\title{
The impact of the COVID-19 pandemic on physical and mental health in the two largest economies in the world: a comparison between the United States and China
}

\author{
Cuiyan Wang ${ }^{1} \cdot$ Connor Tripp $^{2}$ (1) Samuel F. Sears ${ }^{2} \cdot$ Linkang Xu $^{1} \cdot$ Yilin Tan $^{1} \cdot$ Danqing Zhou $^{1} \cdot$ Wenfang Ma $^{1} \cdot$ \\ Ziqi Xu ${ }^{1} \cdot$ Natalie A. Chan ${ }^{3}$ Cyrus Ho ${ }^{4,5} \cdot \operatorname{Roger~Ho}{ }^{5,6}$
}

Received: 19 August 2020 / Accepted: 8 June 2021 / Published online: 14 June 2021

(C) The Author(s), under exclusive licence to Springer Science+Business Media, LLC, part of Springer Nature 2021

\begin{abstract}
The broad impact of the COVID-19 on selfreported daily behaviors and health in Chinese and US samples remains unknown. This study aimed to compare physical and mental health between people from the United States (U.S.) and China, and to correlate mental health parameters with variables relating to physical symptoms, knowledge about COVID-19, and precautionary health behaviors. To minimize risk of exposure, respondents were electronically invited by existing study respondents or by data sourcing software and surveys were completed via online survey platforms. Information was collected on demographics, physical symptoms, contact history, knowledge about COVID-19,
\end{abstract}

Cuiyan Wang and Connor Tripp have contributed equally to this work.

Supplementary Information The online version contains supplementary material available at https://doi.org/10.1007/ s10865-021-00237-7.

Samuel F. Sears

searss@ecu.edu

1 Institute of Cognitive Neuroscience, Faculty of Education, Huaibei Normal University, Huaibei, China

2 Department of Psychology, East Carolina University, Greenville, NC 27858-4353, USA

3 Faculty of Medicine, Dentistry and Health, University of Sheffield, Sheffield, UK

4 Department of Psychological Medicine, National University Health System, Singapore, Singapore

5 Department of Psychological Medicine, Yong Loo Lin School of Medicine, National University of Singapore, Singapore, Singapore

6 Institute of Health Innovation and Technology (iHealthtech), National University of Singapore, Singapore 119228, Singapore psychologic parameters (i.e. IES-R; DASS-21), and health behaviors. The study included a total of 1445 respondents (584 U.S.; 861 China). Overall, Americans reported more physical symptoms, contact history, and perceived likelihood of contracting COVID-19. Americans reported more stress and depressive symptoms, while Chinese reported higher acute-traumatic stress symptoms. Differences were identified regarding face mask use and desires for COVID-19 related health information, with differential mental health implications. Physical symptoms that were possibly COVID-19 related were associated with adverse mental health. Overall, American and Chinese participants reported different mental and physical health parameters, health behaviors, precautionary measures, and knowledge of COVID-19; different risk and protective factors were also identified.

Keywords China C COVID-19 $\cdot$ Masks · Pandemic · Psychological impact · United States

\section{Introduction}

The coronavirus disease 2019 (COVID-19) was declared a pandemic by the World Health Organization (WHO) on March 11, 2020 (Mahase, 2020). China was the first country that identified the novel coronavirus as the cause of the pandemic. As of early-July, the number of confirmed cases was 84,871 , the number of death cases was 4641 and the number of recovered cases was 79,720 in China (John Hopkins University, 2020). Currently, the number of confirmed cases is around 86,829 , the number of death cases is around 4634 , and the number of recovered cases is around 81,886 in China. Strict quarantine and travel restriction measures and medical treatment mobilization were generally successful in reducing the transmission of COVID-19 (Salo, 2020). 
The US has the largest number of COVID-19 cases in the world at the time of writing. By early-July, the number of confirmed cases was $2,888,729$, the number of death cases was 129,947 and the number of recovered cases was 906,763 in the United States (U.S.) (JHU, 2020). Currently, the number of confirmed cases exceeds 17,000,000, the number of death cases exceeds 318,000 , and the number of recovered cases exceeds 43,000,000 in the U.S. There have been some major differences in public response between the U.S. and China. First, wearing face masks in public is an essential component of Chinese and U.S. prevention strategies (Wang et al., 2020b). However, the long-standing air pollution has made face masks an essential accessory among the Chinese, even in normal circumstances (Ho et al., 2014), while maskwearing in the U.S. has typically been low in comparisons of cross-country comparisons (Steelfisher et al., 2010). Second, access to the COVID-19 testing in the U.S. was initially limited. Widespread availability of COVID-19 testing in China, in combination with lockdown and social isolation, appears to have worked to limit community spread and shorten the duration of restrictions on economic activity (Tan et al., 2020a, 2020b).

The lockdown measures and economic recession associated with the COVID-19 pandemic has broadened the impact beyond infectious disease and lifestyle considerations and now includes societal considerations such as stress, mental health, and daily health behaviors. Rapid and significant societal changes had prompted potentially more anxiety and depressive symptoms due to disrupted travel plans, social isolation, information overload about the pandemic and panic buying of necessity goods (Wang et al., 2020b; Tan et al., 2020a, 2020b; Ho et al., 2020a, Wang et al., 2020c, Hao et al., 2020). Recent research indicated that Asian Americans were less likely to report psychiatric symptoms as compared to Caucasian Americans during the COVID-19 pandemic (Liu et al., 2020). However, no other comparisons about the physical and mental health reports of the general population in China and U.S. are available during the COVID-19 pandemic.

There is a research gap in cross-country research during the COVID-19 pandemic (Tran et al., 2020b). As a result, we proposed a novel study to conduct an in-depth and cross-country analysis of the physical and mental health of the American and Chinese. We hypothesized that there were significant differences in mental and physical health parameters, health behaviors, precautionary measures and knowledge of COVID-19. The aims of this study were (a) to compare the levels of physical and mental health parameters between American and Chinese respondents during the pandemic; (b) to correlate psychological impact, depression, anxiety and stress scores with variables relating to physical symptoms, knowledge and concerns about COVID19 and precautionary measures in American and Chinese respondents; (c) identify specific risk and protective factors for mental health in the two largest economies during the pandemic.

\section{Methods}

\section{Study design and study population}

We conducted a cross-cultural study using a cross-sectional design to compare the impact of COVID-19 on physical and mental health outcomes within and between samples recruited from the United States (US) and China. The study was conducted from February 28 to March 1 in China and from April 21 to April 29 in the U.S. when the number of COVID-19 cases peaked in both countries. Respondentdriven sampling strategies focused on recruiting participants from the general public living in the U.S. and China during the COVID-19 pandemic was utilized.

\section{Procedure}

As the Chinese and U.S. Governments recommended the public to minimize face-to-face interaction and isolate themselves during the study period, new respondents were electronically invited by existing study respondents or by data sourcing software. The respondents completed the questionnaires through an online survey platform ('SurveyStar', Changsha Ranxing Science and Technology in China and Amazon Mechanical Turk and Google Forms Online Survey in the U.S.). Participants from both the U.S. and China were required to read a summary of the study and provide informed consent by agreeing to participate with a click on a designated consent box, before proceeding to the full survey. The Institutional Review Board of the East Carolina University (The U.S.) (UMCIRB 20-000838) and the Huaibei Normal University (China) (HBU-IRB-2020-002) approved the studies for each country respectively. All respondents provided informed consent. The collected data were anonymous and treated as confidential.

\section{Outcomes}

This study used the National University of Singapore (NUS) COVID-19 questionnaire, and its psychometric properties had been established in the initial phase and peak of the COVID-19 epidemic (Wang et al., 2020a, 2020b). The NUS COVID-19 questionnaire consisted of questions that covered several areas: (1) demographic data; (2) physical symptoms related to COVID-19 in the past 14 days; (3) contact history with COVID-19 in the past 14 days; (4) knowledge and concerns about COVID-19 and (5) precautionary health behaviors against COVID-19 in the past 14 days. 
From the NUS-COVID 19, demographic data about age, gender, education, household size, marital status, parental status and residential city in the past 14 days were collected. Items reflecting physical symptoms related to COVID-19 were gathered, including cough, fever, gastrointestinal symptoms and other symptoms. Respondents also rated their physical health status and stated their history of chronic medical illness. Health service utilization variables in the past 14 days included consultation with a doctor in the clinic, being quarantined by the health authority and recent testing for COVID-19.

Knowledge and concerns related to COVID-19 included knowledge about the routes of transmission, level of confidence in diagnosis, source and level of satisfaction of health information about COVID-19, the likelihood of contracting and surviving COVID-19 and the number of hours spent on viewing information about COVID-19 per day. Information related to precautionary measures against COVID-19 included avoidance of sharing cutlery during meals, covering mouth when coughing and sneezing, various measures of hand hygiene and wearing a face mask regardless of the presence or absence of symptoms was surveyed. The respondents were asked the average number of hours staying at home per day during the COVID-19 pandemic. Respondents were also asked whether they felt unnecessary worry had been made about the COVID-19 pandemic. On this COVID-19 specific measure, respondents were also asked about perceptions of discrimination from other countries as a result of COVID-19.

The psychological impact of COVID-19 was measured using the Impact of Event Scale-Revised (IES-R). The IES-R is a self-administered questionnaire that has been well-validated in the American and Chinese population for determining the extent of psychological impact after exposure to a traumatic event (i.e., the COVID-19 pandemic) within one week of exposure (Chew et al., 2020; Hosey et al., 2019; Zhang et al., 2014a). This 22-item questionnaire which is composed of three subscales, aims to measure the mean avoidance, intrusion and hyperarousal (Zhang et al., 2014b). The total IES-R score is divided into 0-23 (normal), 24-32 (mild psychological impact), 33-36 (moderate psychological impact) and $>37$ (severe psychological impact) (Creamer et al., 2003). The total IES-R score $>24$ suggests the presence of post-traumatic stress disorder (PTSD) symptoms (Lee et al., 2018). In this study, the Cronbach's alpha for the Chinese and U.S. versions of IES-R are as follows: China $(\alpha=0.949)$ and U.S. $(\alpha=0.959)$.

The mental health status of respondents was measured using the Depression, Anxiety and Stress Scale (DASS21 ) and calculation and interpretation of total and subscale scores were based on a previous study (Le et al., 2019). DASS-21 has been used to assess mental health in Chinese (Ho et al., 2020a, 2020b; Quek et al., 2018) and American
(Norton, 2009) samples. In this study, the Cronbach's alpha for the Chinese and U.S. versions of the DASS-21 are as follows: China: stress $(\alpha=0.888)$, anxiety $(\alpha=0.845)$, depression $(\alpha=0.878)$ and U.S.: stress $(\alpha=0.921)$, anxiety ( $\alpha=0.914)$, and depression $(\alpha=0.938)$. IES-R and DASS-21 were previously used in research related to the COVID-19 epidemic (Chew et al., 2020; Hao et al., 2020; Tan et al., 2020a, 2020b; Wang et al., 2020a).

\section{Statistical analysis}

Descriptive statistics were calculated for demographic characteristics, physical symptoms and health service utilization, contact history, knowledge and concern, precautionary measure and additional health information variables. To analyze the differences in the levels of psychological impact, levels of depression, anxiety and stress, the independent sample t-test was used to compare the mean score between the American and Chinese respondents. The chi-squared test was used to analyze the differences in categorical variables between the two samples. Given that there were multiple comparisons between two countries, adjusted $p$ values based on the Bonferroni correction were applied. We used linear regressions to calculate the univariate associations between independent and dependent variables including the IES-S score and DASS-21 stress, anxiety and depression subscale scores for the American and Chinese respondents separately. All tests were two-tailed, with a significance level of $p<0.05$. Statistical analysis was performed on SPSS Statistic 21.0.

\section{Results}

\section{Comparison between the American and Chinese respondents and their mental health status}

For the American sample, we received responses from 1100 respondents, and 516 respondents did not complete the questionnaires or were removed from the study due to inappropriate responses to validity items (e.g., Participants who responded "rarely," "sometimes," "frequently," or "always," rather than "never" on items such as "How often do you travel to Neptune for lunch?" were removed). This sampling strategy helped detect likely random responders. The final sample included 584 respondents in the U.S. who had completed the questionnaires $(53.00 \%)$. For the China sample, we excluded 4 incomplete questionnaires, which left 861 of a total of $865(99.53 \%)$ valid questionnaires in China. Thus, there were 584 participants in the U.S. sample and 861 in the Chinese sample for a total of 1445 participants in the study. Notably, for comparative analyses, adjusted $p$ values based on the Bonferroni correction were applied, as 
a statistical means of controlling error rate given the large number of variables and comparisons.

Figure 1 compares the mean scores of DASS- 21 stress, anxiety and depression subscales and IES-R scores between American and Chinese respondents. For the DASS-21 stress subscale $\left(M_{\text {China }}=7.86, S D_{\text {China }}=7.93 ; M_{U S A}=9.28\right.$, $\left.S D_{U S A}=9.71\right)$, American respondents reported significantly higher scores than Chinese respondents $(\mathrm{t}=2.93, p<0.01$, 95\% CI 0.47 to 2.37 ). For the DASS-21 depression subscale $\left(M_{\mathrm{China}}=6.38, S D_{\mathrm{China}}=7.39 ; M_{\mathrm{USA}}=8.33, S D_{\mathrm{USA}}=10.19\right)$, American respondents reported significantly higher depression scores ( $\mathrm{t}=3.97, p<0.001,95 \%$ CI 0.98 to 2.91$)$. For the DASS-2 1 anxiety subscale $\left(M_{\text {China }}=6.15, S D_{\text {China }}=6.94\right.$; $M_{\mathrm{USA}}=5.70, S D_{\mathrm{USA}}=8.54$ ), there were no significant difference between respondents in the two countries $(\mathrm{t}=1.05$, $p>0.05,95 \%$ CI -1.28 to 0.39$)$.

For IES-R $\left(M_{\text {China }}=30.76, S D_{\text {China }}=16.34 ; M_{\mathrm{USA}}=22.19\right.$, $\left.S D_{\text {USA }}=16.34\right)$, Chinese respondents had significantly higher scores than American respondents $(\mathrm{t}=9.06$, $p<0.001,95 \%$ CI -10.42 to -6.71$)$. The mean IES-R scores of Chinese respondents was higher than 24 points, suggesting the presence of clinically significant PTSD symptoms in Chinese respondents.

\section{Demographic characteristics and their association with psychological impact and adverse mental health status}

The majority of American respondents were women (52.9\%), having a household size of 3-5 people (46.6\%), well educated (87.9\% with a bachelor or higher degree) and married (44.8\%). Similarly, the majority of Chinese respondents were women (75\%), having a household size of 3-5 people $(80.4 \%)$ and well educated $(87.6 \%$ with a bachelor or higher degree). In comparison to American respondents, there was a significantly higher proportion of Chinese respondents who

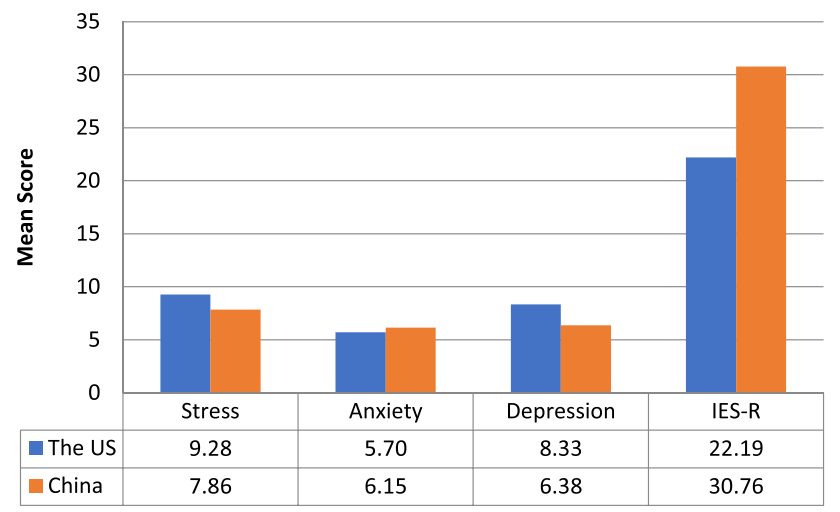

Fig. 1 Comparison of the mean scores of DASS-stress, anxiety and depression subscales as well as IES-R scores between American and Chinese respondents were female $(75 \%, p<0.001)$, younger $(21.4-30.8$ years: $46.5 \%, p<0.005)$, married $(83.5 \%, p<0.017)$, had a child older than 16 years $(51.1 \%, p<0.017)$, had a household size of $3-5$ people $(80.4 \%, p<0.008)$, had lower education level (junior high school and primary school: $7.1 \%, p<0.08$ ) and reported student status $(62.8 \%, p<0.005)$. (See Supplementary Table 1 for more detailed comparisons of demographic information).

Linear regression analysis was used to assess associations between demographic factors and scores on IES-R and DASS-21 to identify potential risk and protective factors for mental health in response to COVID-19. For American respondents, age younger than 49.6 years was a risk factor significantly associated with the higher scores of IES-R and all DASS-21 subscales $(p<0.01)$, while having a child older than 16 years of age and student status were protective factors significantly associated with the lower score of IES-R and all DASS-21 subscales $(p<0.05)$ (see Table 1). For Chinese respondents, male gender was significantly associated with a lower score of IES-R, but higher DASS-21 depression scores $(p<0.01)$. Notwithstanding, there were other differences between American and Chinese respondents.

\section{Physical symptoms, health status and its association with psychological impact and adverse mental health status}

For physical symptoms resembling COVID-19 and health status, there was a significantly higher proportion of American respondents who reported fever, cough, breathing difficulty, sore throat, gastrointestinal symptoms, recent consultation with a doctor, recent COVID-19 testing, recent quarantine, poor self-rating health status and history of chronic illness $(p<0.01)$. (see Supplementary Table 2 for comparisons of physical symptoms between Chinese and American respondents). Nevertheless, there was a significantly higher proportion of Chinese respondents who reported myalgia, coryza and coverage by medical insurance as compared with American respondents $(p<0.01)$.

Linear regression showed that headache and dizziness were significantly associated with higher IES-R scores and DASS-21 subscale scores in both countries $(p<0.05)$ (see Table 2). Similarly, cough, difficulty in breathing, gastrointestinal symptoms and recent quarantine were significantly associated with higher DASS-21 subscale scores in both countries $(p<0.01)$. Persistent fever, chills and coryza were significantly associated with DASS-21 stress and depression subscale scores $(p<0.05)$, while very poor health status was significantly associated with DASS-21 depression score $(p<0.001)$ in both countries. Sore throat was associated with DASS-21 subscale scores $(p<0.001)$ in Chinese respondents only. 
Table 1 Comparison of the association between demographic variables and the psychological impact as well as adverse mental health status between American and Chinese respondents (U.S. $n=584$, China $\mathrm{n}=861$, Total $\mathrm{N}=1445$ )

\begin{tabular}{|c|c|c|c|c|c|c|c|c|}
\hline \multirow{3}{*}{$\begin{array}{l}\text { Demo- } \\
\text { graphic } \\
\text { character- } \\
\text { istics }\end{array}$} & \multicolumn{8}{|l|}{ The US } \\
\hline & \multicolumn{2}{|c|}{ Impact of event } & \multicolumn{2}{|l|}{ Stress } & \multicolumn{2}{|l|}{ Anxiety } & \multicolumn{2}{|l|}{ Depression } \\
\hline & $B$ & $t$ & $B$ & $t$ & $B$ & $t$ & $B$ & $t$ \\
\hline \multicolumn{9}{|l|}{ Gender } \\
\hline Male & -0.08 & -0.82 & $-\mathrm{s} 0.07$ & -0.87 & -0.02 & -0.19 & 0.03 & 0.29 \\
\hline Female & Reference & & Reference & & Reference & & Reference & \\
\hline \multicolumn{9}{|l|}{ Age (years) } \\
\hline$[12-21.4]$ & 1.35 & $3.94 * * * \prime$ & 0.85 & $3.15^{* * \prime}$ & 1.44 & $3.67 * * * \prime$ & 1.13 & $3.14 * * \prime$ \\
\hline (21.4-30.8] & 0.69 & $4.98 * * * \prime$ & 0.54 & $4.90 * * * \prime$ & 1.01 & $6.34 * * * \prime$ & 0.71 & $4.89 * * * \prime$ \\
\hline$(30.8-40.2]$ & 0.51 & $3.85 * * * \prime$ & 0.33 & $3.12 * * \prime$ & 0.55 & $3.65 * * * \prime$ & 0.59 & $4.19 * * * \prime$ \\
\hline (40.2-49.6] & 0.25 & 1.66 & 0.08 & 0.71 & 0.21 & 0.71 & 0.32 & 2.07 \\
\hline$>49.6$ & Reference & & Reference & & Reference & & Reference & \\
\hline \multicolumn{9}{|c|}{ Parental status } \\
\hline $\begin{array}{l}\text { Has a child } \\
\text { who is } \\
\text { above or } \\
\text { below } \\
16 \text { years } \\
\text { old }\end{array}$ & 0.11 & 0.90 & -0.01 & -0.14 & -0.06 & -0.39 & -0.06 & -0.45 \\
\hline $\begin{array}{l}\text { Has a child } \\
\text { who is } \\
\text { above } \\
16 \text { years } \\
\text { old }\end{array}$ & -0.39 & $-2.63 * *$ & -0.27 & $-2.42 *$ & -0.44 & $-2.64 * *$ & -0.45 & $-3.00^{* *}$ \\
\hline No children & Reference & & Reference & & Reference & & Reference & \\
\hline \multicolumn{9}{|c|}{ Household size } \\
\hline $\begin{array}{l}6 \text { people or } \\
\text { more }\end{array}$ & -0.11 & -0.37 & 0.07 & 0.31 & 0.06 & 0.19 & -0.15 & -0.48 \\
\hline 3-5 people & 0.33 & 2.33 & 0.21 & 1.90 & 0.22 & 1.36 & 0.32 & 2.17 \\
\hline 2 people & 0.03 & 0.17 & 0.11 & 0.90 & 0.09 & 0.52 & 0.07 & 0.42 \\
\hline 1 person & Reference & & Reference & & Reference & & Reference & \\
\hline \multicolumn{9}{|c|}{ Educational level } \\
\hline $\begin{array}{l}\text { High } \\
\text { School }\end{array}$ & -0.12 & -0.24 & -0.35 & -0.86 & -0.60 & -0.99 & -0.03 & -0.06 \\
\hline $\begin{array}{l}\text { University } \\
\text { (Bach- } \\
\text { elor's) }\end{array}$ & -0.23 & -0.45 & -0.40 & -1.00 & -0.36 & -0.62 & -0.19 & -0.35 \\
\hline $\begin{array}{l}\text { University } \\
\text { (Masters } \\
\text { or doctor) }\end{array}$ & -0.06 & -0.11 & -0.36 & -0.90 & -0.36 & -0.61 & -0.19 & -0.36 \\
\hline $\begin{array}{l}\text { Second- } \\
\text { ary and } \\
\text { below }\end{array}$ & Reference & & Reference & & Reference & & Reference & \\
\hline \multicolumn{9}{|c|}{ Employment status } \\
\hline Student & -0.59 & $-3.03 * *^{\prime}$ & -0.41 & $-2.69 * \prime$ & -0.68 & $-3.01 * \prime$ & -0.54 & $-2.62 * \prime$ \\
\hline $\begin{array}{l}\text { Unem- } \\
\text { ployed }\end{array}$ & 0.38 & 1.69 & 0.30 & 1.71 & 0.46 & 1.75 & 0.37 & 1.58 \\
\hline Housewife & 0.30 & 1.67 & 0.48 & $3.37 * * \prime$ & 0.14 & 0.66 & 0.38 & 2.00 \\
\hline Retired & 0.01 & 0.04 & 0.12 & 0.52 & -0.14 & -0.40 & -0.10 & -0.33 \\
\hline Employed & Reference & & Reference & & Reference & & Reference & \\
\hline
\end{tabular}


Table 1 (continued)

\begin{tabular}{|c|c|c|c|c|c|c|c|c|c|c|c|}
\hline \multirow{3}{*}{$\begin{array}{l}\text { Demo- } \\
\text { graphic } \\
\text { character- } \\
\text { istics }\end{array}$} & \multicolumn{11}{|l|}{ The US } \\
\hline & \multicolumn{3}{|l|}{ Impact of event } & \multicolumn{2}{|c|}{ Stress } & \multicolumn{2}{|c|}{ Anxiety } & \multicolumn{4}{|c|}{ Depression } \\
\hline & \multirow[t]{2}{*}{$B$} & \multicolumn{2}{|l|}{$t$} & $B$ & \multirow[t]{2}{*}{$t$} & \multirow[t]{2}{*}{$B$} & $t$ & \multicolumn{2}{|l|}{$B$} & \multicolumn{2}{|r|}{$t$} \\
\hline \multicolumn{9}{|c|}{ Marital status } & & & \\
\hline Single & 0.50 & \multicolumn{2}{|c|}{1.32} & 0.18 & 0.60 & 0.22 & 0.51 & \multicolumn{2}{|c|}{0.53} & & 1.36 \\
\hline Married & 0.39 & \multicolumn{2}{|c|}{1.04} & 0.04 & 0.14 & -0.03 & -0.07 & 0. & & & 0.77 \\
\hline $\begin{array}{l}\text { Divorced } \\
\text { or sepa- } \\
\text { rated }\end{array}$ & 0.38 & & & -0.08 & -0.25 & -0.29 & -0.60 & 0 . & & & 0.24 \\
\hline Widowed & Reference & & & Reference & & Refer & & & rence & & \\
\hline Demograph & c characteristics & China & & & & & & & & & \\
\hline & & Impact o & event & Stress & & & Anxiety & & Depress & & \\
\hline & & $B$ & $t$ & $B$ & $t$ & & $B$ & $t$ & $B$ & $t$ & \\
\hline Gender & & & & & & & & & & & \\
\hline Male & & -0.26 & $-2.61 * *$ & 0.08 & 1.38 & & 0.18 & 1.90 & 0.22 & $2.89 * *$ & \\
\hline Female & & Referenc & & Referer & & & Reference & & Referen & & \\
\hline Age (years) & & & & & & & & & & & \\
\hline [12-21.4] & & 0.77 & 2.28 & -0.03 & -0.16 & & 0.29 & 0.92 & -0.02 & -0.07 & \\
\hline$(21.4-30.8]$ & & 0.59 & 1.75 & 0.02 & 0.08 & & 0.36 & 1.17 & 0.09 & 0.36 & \\
\hline$(30.8-40.2]$ & & 0.63 & 1.62 & -0.03 & -0.15 & & 0.29 & 0.80 & 0.03 & 0.12 & \\
\hline$(40.2-49.6]$ & & 0.26 & 0.70 & -0.15 & -0.70 & & -0.02 & -0.05 & -0.18 & -0.63 & \\
\hline$>49.6$ & & Referenc & & Referer & & & Reference & & Referen & & \\
\hline Parental stc & & & & & & & & & & & \\
\hline $\begin{array}{r}\text { Has a child } \\
\text { below } 16\end{array}$ & $\begin{array}{l}\text { who is above or } \\
\text { ears old }\end{array}$ & 0.12 & 0.94 & 0.03 & 0.34 & & 0.11 & 0.87 & 0.09 & 0.88 & \\
\hline $\begin{array}{c}\text { Has a child } \\
16 \text { years }\end{array}$ & $\begin{array}{l}\text { who is above } \\
\text { Id }\end{array}$ & 0.10 & 0.96 & 0.003 & 0.06 & & -0.03 & -0.34 & -0.02 & -0.29 & \\
\hline No children & & Referenc & & Referer & & & Reference & & Referen & & \\
\hline Household & & & & & & & & & & & \\
\hline 6 people or & more & 1.44 & 2.20 & 0.50 & 1.32 & & 0.84 & 1.40 & 0.12 & 0.24 & \\
\hline 3-5 people & & 1.32 & 2.04 & 0.45 & 1.19 & & 0.77 & 1.29 & 0.06 & 0.13 & \\
\hline 2 people & & 1.19 & 1.76 & 0.44 & 1.12 & & 0.61 & 0.99 & -0.16 & -0.31 & \\
\hline 1 person & & Referenc & & Referer & & & Reference & & Referen & & \\
\hline Educationa & level & & & & & & & & & & \\
\hline High Schoo & & -0.06 & -0.22 & 0.19 & 1.29 & & 0.25 & 1.07 & 0.16 & 0.81 & \\
\hline University & Bachelor's) & 0.33 & 1.93 & 0.06 & 0.62 & & 0.04 & 0.26 & -0.01 & -0.09 & \\
\hline University & Masters or doctor) & 0.24 & 1.18 & 0.13 & 1.11 & & 0.24 & 1.31 & 0.14 & 0.89 & \\
\hline Secondary & nd below & Referenc & & Referer & & & Reference & & Referen & & \\
\hline Employmer & status & & & & & & & & & & \\
\hline Student & & -0.63 & -0.85 & -0.12 & -0.27 & & -0.75 & -1.10 & -0.19 & -0.34 & \\
\hline Unemploye & & 0.06 & 0.30 & 0.10 & 0.91 & & 0.29 & 1.67 & 0.32 & 2.25 & \\
\hline Housewife & & -0.52 & -1.99 & -0.03 & -0.17 & & 0.10 & 0.40 & 0.10 & 0.47 & \\
\hline Retired & & -0.16 & -1.64 & -0.01 & -0.20 & & -0.01 & -0.10 & 0.06 & 0.74 & \\
\hline Employed & & Referen & & Referer & & & Reference & & Referen & & \\
\hline Marital sta & & & & & & & & & & & \\
\hline Single & & 1.06 & 1.41 & 0.41 & 0.94 & & 0.60 & 0.87 & 0.48 & 0.84 & \\
\hline Married & & 1.27 & 1.71 & 0.46 & 1.06 & & 0.80 & 1.17 & 0.58 & 1.02 & \\
\hline Divorced o & separated & 1.27 & 1.35 & 0.60 & 1.10 & & 1.00 & 1.16 & 0.60 & 0.84 & \\
\hline Widowed & & Referen & & Referer & & & Reference & & Referen & & \\
\hline
\end{tabular}

${ }^{*} p<0.05 ; * *<0.01 ; * * * p 0.001$ (significance level was corrected by Bonferroni's method, ${ }^{* \prime} p<0.0125 ; *^{*} p<0.0025 ; *^{* *} p<0.00025$ ) 
Table 2 Comparison of physical symptoms related to COVID-19, health status and contact history between American and Chinese respondents (U.S. $\mathrm{n}=584$, China $\mathrm{n}=861$, Total $\mathrm{N}=1445$ )

\begin{tabular}{|c|c|c|c|c|c|c|c|c|}
\hline \multirow{3}{*}{$\begin{array}{l}\text { Symptoms and } \\
\text { physical health } \\
\text { status }\end{array}$} & \multicolumn{8}{|l|}{ The US } \\
\hline & \multicolumn{2}{|c|}{ Impact of event } & \multicolumn{2}{|l|}{ Stress } & \multicolumn{2}{|l|}{ Anxiety } & \multicolumn{2}{|l|}{ Depression } \\
\hline & $B$ & $t$ & $B$ & $t$ & $B$ & $t$ & $B$ & $t$ \\
\hline \multicolumn{9}{|l|}{ Persistent fever } \\
\hline Yes & 0.29 & 0.57 & 0.88 & $2.22 *$ & 0.72 & 1.25 & 1.18 & $2.26^{*}$ \\
\hline No & \multicolumn{2}{|l|}{ Reference } & \multicolumn{2}{|l|}{ Reference } & \multicolumn{2}{|l|}{ Reference } & \multicolumn{2}{|l|}{ Reference } \\
\hline \multicolumn{9}{|l|}{ Chills } \\
\hline Yes & 0.41 & 1.25 & 0.60 & $2.29 *$ & 0.73 & 1.92 & 0.76 & $2.20 *$ \\
\hline No & \multicolumn{2}{|l|}{ Reference } & \multicolumn{2}{|l|}{ Reference } & \multicolumn{2}{|l|}{ Reference } & \multicolumn{2}{|l|}{ Reference } \\
\hline \multicolumn{9}{|l|}{ Headaches } \\
\hline Yes & 0.38 & $2.79 * *$ & 0.41 & $3.84 * * *$ & 0.47 & $2.99 * *$ & 0.42 & $2.91 * *$ \\
\hline No & \multicolumn{2}{|l|}{ Reference } & \multicolumn{2}{|l|}{ Reference } & \multicolumn{2}{|l|}{ Reference } & \multicolumn{2}{|l|}{ Reference } \\
\hline Myalgia & & & & & & & & \\
\hline Yes & 0.99 & $2.13^{*}$ & 0.37 & 1.01 & 1.09 & $2.02 *$ & 0.89 & 1.84 \\
\hline No & Reference & & Reference & & Reference & & Reference & \\
\hline Cough & & & & & & & & \\
\hline Yes & 0.41 & $2.38^{*}$ & 0.45 & $3.28 * *$ & 0.48 & $2.40 *$ & 0.49 & $2.70 * *$ \\
\hline No & Reference & & Reference & & Reference & & Reference & \\
\hline Difficulty breath & ing & & & & & & & \\
\hline Yes & 0.41 & 1.40 & 0.55 & $2.39 *$ & 0.91 & $2.70 * *$ & 0.63 & $2.07 *$ \\
\hline No & Reference & & Reference & & Reference & & Reference & \\
\hline Dizziness & & & & & & & & \\
\hline Yes & 0.73 & $2.43 *$ & 0.98 & $4.20 * * *$ & 1.50 & $4.36 * * *$ & 1.20 & $3.87 * * *$ \\
\hline No & Reference & & Reference & & Reference & & Reference & \\
\hline Coryza & & & & & & & & \\
\hline Yes & 1.12 & 0.91 & 2.37 & $2.47 *$ & -0.79 & -0.55 & 3.17 & $2.49 *$ \\
\hline No & Reference & & Reference & & Reference & & Reference & \\
\hline Sore throat & & & & & & & & \\
\hline Yes & -0.13 & -0.66 & -0.02 & -0.10 & -0.05 & -0.22 & 0.13 & 0.63 \\
\hline No & Reference & & Reference & & Reference & & Reference & \\
\hline Persistent fever & with cough o & r difficult & & & & & & \\
\hline Yes & 0.12 & 0.19 & 0.87 & 1.81 & 0.72 & 1.02 & 1.43 & $2.24 *$ \\
\hline No & Reference & & Reference & & Reference & & Reference & \\
\hline Nausea \& vomit & ing \& diarrh & & & & & & & \\
\hline Yes & 0.08 & 0.31 & 0.47 & $2.34 *$ & 0.70 & $2.39 *$ & 0.91 & $3.46 * *$ \\
\hline No & Reference & & Reference & & Reference & & Reference & \\
\hline Have you been $t$ & o an outpatie & ent clinic & 14 days & & & & & \\
\hline Yes & 0.32 & 1.69 & 0.23 & 1.53 & 0.43 & 1.95 & 0.33 & 1.65 \\
\hline No & Reference & & Reference & & Reference & & Reference & \\
\hline Have you done & ovel Corona & ivirus test & t 14 days & & & & & \\
\hline Yes & 0.89 & $2.90 * *$ & 0.70 & $2.88^{* *}$ & 1.44 & $4.08 * * *$ & 0.88 & $2.73 * *$ \\
\hline No & Reference & & Reference & & Reference & & Reference & \\
\hline Have you been & uarantined $i$ & in the pas & & & & & & \\
\hline Yes & 0.58 & $3.34 * *$ & 0.30 & $2.20^{*}$ & 0.85 & $4.27 * * *$ & 0.39 & $2.14 *$ \\
\hline No & Reference & & Reference & & Reference & & Reference & \\
\hline Self-rate health & status & & & & & & & \\
\hline Very poor & 0.15 & 1.01 & 0.29 & $2.41^{* \prime}$ & 0.24 & 1.38 & 0.62 & $4.01 * * * \prime$ \\
\hline Poor & 0.08 & 0.71 & -0.09 & -1.02 & -0.05 & -0.39 & -0.03 & -0.22 \\
\hline
\end{tabular}


Table 2 (continued)

\begin{tabular}{|c|c|c|c|c|c|c|c|c|}
\hline \multirow{3}{*}{$\begin{array}{l}\text { Symptoms and } \\
\text { physical health } \\
\text { status }\end{array}$} & \multicolumn{8}{|l|}{ The US } \\
\hline & \multicolumn{2}{|c|}{ Impact of event } & \multicolumn{2}{|l|}{ Stress } & \multicolumn{2}{|c|}{ Anxiety } & \multicolumn{2}{|c|}{ Depression } \\
\hline & $B$ & $t$ & $B$ & $t$ & $B$ & $t$ & $B$ & $t$ \\
\hline $\begin{array}{l}\text { Good or very } \\
\text { good }\end{array}$ & \multicolumn{2}{|l|}{ Reference } & \multicolumn{2}{|l|}{ Reference } & \multicolumn{2}{|c|}{ Reference } & \multicolumn{2}{|c|}{ Reference } \\
\hline \multicolumn{9}{|c|}{ Medical insurance } \\
\hline Yes & -0.03 & -0.18 & -0.09 & -0.79 & -0.10 & -0.58 & -0.1 & -0.92 \\
\hline No & \multicolumn{2}{|l|}{ Reference } & \multicolumn{2}{|l|}{ Reference } & \multicolumn{2}{|c|}{ Reference } & \multicolumn{2}{|c|}{ Reference } \\
\hline \multicolumn{9}{|l|}{ Chronic illness } \\
\hline Yes & -0.01 & -0.09 & 0.14 & 1.42 & -0.05 & -0.33 & 0.21 & 1.60 \\
\hline No & \multicolumn{2}{|l|}{ Reference } & \multicolumn{2}{|l|}{ Reference } & \multicolumn{2}{|c|}{ Reference } & \multicolumn{2}{|c|}{ Reference } \\
\hline \multirow{3}{*}{$\begin{array}{l}\text { Symptoms and } \\
\text { physical health } \\
\text { status }\end{array}$} & China & & & & & & & \\
\hline & Impact of e & event & Stress & & Anxiet & & Depres & \\
\hline & $B$ & $t$ & $B$ & $t$ & $B$ & $t$ & $B$ & $t$ \\
\hline Persistent fever & & & & & & & & \\
\hline Yes & 1.43 & 1.11 & 3.55 & $4.81 * * *$ & 3.24 & $2.75 * *$ & 3.44 & $3.56 * * *$ \\
\hline No & Reference & & Reference & & Refere & & Refere & \\
\hline Chills & & & & & & & & \\
\hline Yes & 0.69 & 1.84 & 0.90 & $4.16^{* * *}$ & 0.83 & $2.42 *$ & 0.87 & $3.08 * *$ \\
\hline No & Reference & & Reference & & Refere & & Refere & \\
\hline Headaches & & & & & & & & \\
\hline Yes & 0.59 & $3.02 * *$ & 0.46 & $4.07 * * *$ & 0.76 & $4.26 * * *$ & 0.51 & $3.45^{* *}$ \\
\hline No & Reference & & Reference & & Refere & & Refere & \\
\hline Myalgia & & & & & & & & \\
\hline Yes & 0.50 & $2.83 * *$ & 0.42 & $4.13 * * *$ & 0.59 & $3.67 * * *$ & 0.58 & $4.42 * * *$ \\
\hline No & Reference & & Reference & & Refere & & Refere & \\
\hline Cough & & & & & & & & \\
\hline Yes & 0.48 & 1.95 & 0.61 & $4.25 * * *$ & 0.69 & $3.03 * *$ & 0.68 & $3.64 * * *$ \\
\hline No & Reference & & Reference & & Refere & & Refere & \\
\hline Difficulty breath & ing & & & & & & & \\
\hline Yes & 0.77 & 1.46 & 1.06 & $3.47 * *$ & 1.08 & $2.23 *$ & 1.45 & $3.66 * * *$ \\
\hline No & Reference & & Reference & & Refere & & Refere & \\
\hline Dizziness & & & & & & & & \\
\hline Yes & 0.98 & $4.20 * * *$ & 0.80 & $6.00 * * *$ & 0.95 & $4.42 * * *$ & 0.66 & $3.72 * * *$ \\
\hline No & Reference & & Reference & & Refere & & Refere & \\
\hline Coryza & & & & & & & & \\
\hline Yes & 0.66 & $3.40 * *$ & 0.33 & $2.90 * *$ & 0.52 & $2.94 * *$ & 0.53 & $3.64 * * *$ \\
\hline No & Reference & & Reference & & Refere & & Refere & \\
\hline Sore throat & & & & & & & & \\
\hline Yes & 0.35 & 1.50 & 0.60 & $4.53 * * *$ & 0.80 & $3.77 * * *$ & 0.75 & $4.31 * * *$ \\
\hline No & Reference & & Reference & & Refere & & Refere & \\
\hline Persistent fever & with cougho & $r$ difficult & & & & & & \\
\hline Yes & 1.43 & 1.11 & 3.55 & $4.81 * * *$ & 3.24 & $2.75 * *$ & 3.44 & $3.56 * * *$ \\
\hline No & Reference & & Reference & & Refere & & Refere & \\
\hline Nausea \& vomit & ing \& diarrh & & & & & & & \\
\hline Yes & 0.72 & 1.48 & 1.27 & $4.54 * * *$ & 1.39 & $3.11 * *$ & 1.31 & $3.56 * * *$ \\
\hline No & Reference & & Reference & & Refere & & Refere & \\
\hline Have you been $t$ & o an outpatic & ent clinic & 14 days & & & & & \\
\hline Yes & 0.44 & 1.21 & 0.25 & 1.17 & 0.39 & 1.20 & 0.29 & 1.07 \\
\hline
\end{tabular}


Table 2 (continued)

\begin{tabular}{|c|c|c|c|c|c|c|c|c|}
\hline \multirow{3}{*}{$\begin{array}{l}\text { Symptoms and } \\
\text { physical health } \\
\text { status }\end{array}$} & \multicolumn{8}{|l|}{ China } \\
\hline & \multicolumn{2}{|c|}{ Impact of event } & \multicolumn{2}{|l|}{ Stress } & \multicolumn{2}{|c|}{ Anxiety } & \multicolumn{2}{|c|}{ Depression } \\
\hline & $B$ & $t$ & $B$ & $t$ & $B$ & $t$ & $B$ & $t$ \\
\hline No & \multicolumn{2}{|l|}{ Reference } & \multicolumn{2}{|c|}{ Reference } & \multicolumn{2}{|c|}{ Reference } & \multicolumn{2}{|c|}{ Reference } \\
\hline \multicolumn{9}{|c|}{ Have you done novel Coronavirus test in the past 14 days } \\
\hline Yes & -0.32 & -0.50 & -0.20 & -0.54 & -0.02 & -0.03 & -0.06 & -0.12 \\
\hline No & \multicolumn{2}{|l|}{ Reference } & \multicolumn{2}{|c|}{ Reference } & \multicolumn{2}{|c|}{ Reference } & \multicolumn{2}{|c|}{ Reference } \\
\hline \multicolumn{9}{|c|}{ Have you been quarantined in the past 14 days } \\
\hline Yes & 0.35 & 1.73 & 0.25 & $2.14 *$ & 0.55 & $2.94 * *$ & 0.39 & $2.52 *$ \\
\hline No & \multicolumn{2}{|l|}{ Reference } & \multicolumn{2}{|c|}{ Reference } & \multicolumn{2}{|c|}{ Reference } & \multicolumn{2}{|c|}{ Reference } \\
\hline \multicolumn{9}{|c|}{ Self-rate health status } \\
\hline Very poor & 1.57 & $3.24 * * \prime$ & 1.20 & $4.30 * * * \prime$ & 1.67 & $3.78 * * * \prime$ & 1.29 & $3.56 * * * \prime$ \\
\hline Poor & 0.38 & $4.05^{* * * \prime}$ & 0.22 & $4.06 * * * \prime$ & 0.41 & $4.82 * * * \prime$ & 0.38 & $5.51 * * * \prime$ \\
\hline $\begin{array}{l}\text { Good or very } \\
\text { good }\end{array}$ & \multicolumn{2}{|l|}{ Reference } & \multicolumn{2}{|c|}{ Reference } & \multicolumn{2}{|c|}{ Reference } & \multicolumn{2}{|c|}{ Reference } \\
\hline \multicolumn{9}{|c|}{ Medical insurance } \\
\hline Yes & 0.02 & 0.13 & -0.17 & -1.89 & -0.34 & $-2.43^{*}$ & -0.21 & -1.79 \\
\hline No & \multicolumn{2}{|l|}{ Reference } & \multicolumn{2}{|c|}{ Reference } & \multicolumn{2}{|c|}{ Reference } & \multicolumn{2}{|c|}{ Reference } \\
\hline \multicolumn{9}{|l|}{ Chronic illness } \\
\hline Yes & 0.45 & $2.25 *$ & 0.24 & $2.02 *$ & 0.25 & 1.34 & 0.27 & 1.76 \\
\hline No & \multicolumn{2}{|l|}{ Reference } & \multicolumn{2}{|c|}{ Reference } & \multicolumn{2}{|c|}{ Reference } & \multicolumn{2}{|c|}{ Reference } \\
\hline
\end{tabular}

${ }^{*} p<0.05,{ }^{*} p<0.01,{ }^{* * *} p<0.001$ (significance level was corrected by Bonferroni's method, ${ }^{*} p<0.025,{ }^{* * \prime} p<0.005, * * *^{\prime} p<0.0005$ )

\section{Knowledge and concerns about COVID-19 and its association with psychological impact and adverse mental health status}

American and Chinese respondents held significantly different views in terms of knowledge and concerns related to COVID-19 (see Supplementary Table 3 for statistical comparisons of knowledge and concerns related to COVID-19 between Chinese and American respondents). For the routes of transmission, there were significantly more American respondents who agreed that the COVID-19 was transmitted by droplets, contact via contaminated objects, and airborne transmission $(p<0.001)$. For the detection and risk of contracting COVID-19, there were significantly more Chinese respondents who were confident about their doctor's ability to diagnose COVID-19 $(p<0.05)$, perceived greater likelihood of contracting COVID-19 $(p<0.005)$, and felt uncertain about survival during the COVID-19 infection $(p<0.005)$. There were significantly more American respondents who were worried about their family members contracting COVID-19 $(p<0.005)$ and reported contact with patients and materials infected by COVID-19 $(p<0.001)$.

For health information, there were significantly more Chinese who were satisfied with the amount of health information $(p<0.008)$. There were significantly more Chinese respondents who felt discriminated against by other countries $(p<0.001)$. Significantly more American respondents spent more than $5 \mathrm{~h}$ per day to monitor health information regarding the pandemic $(p<0.001)$. Chinese respondents preferred to use the Internet while American respondents preferred to use television to obtain health information $(p<0.017)$.

Linear regression analysis showed that the American and Chinese respondents showed different findings with regards to routes of transmission and perceptions of likelihood of contracting and surviving the virus (see Table 3). American respondents who responded positively on three items related to transmission of the virus, specifically the items that identified droplets, contact with contaminated surfaces and airborne as mode of transmission, reported higher perceived likelihood of contracting COVID-19, and felt discriminated against by other countries due to COVID-19 had higher IES-R or DASS-21 subscale scores $(p<0.05)$. Interestingly, Chinese respondents who were satisfied with health information on COVID-19 had lower IES-R and DASS-21 scores $(p<0.01)$. In terms of mode of communication of health information, Internet was associated with lower IES-R and DASS-21 subscale scores in American respondents $(p<0.01)$ and television was associated with lower DASS-21 subscale scores in American respondents, while radio was associated with higher DASS-21 subscale scores in Chinese respondents $(p<0.05)$. 
Table 3 Comparison of the association of knowledge and concerns related to Covid-19, psychological impact and mental health status in American and Chinese respondents (U.S. $\mathrm{n}=584$, China $\mathrm{n}=861$, Total $\mathrm{N}=1445$ )

\begin{tabular}{|c|c|c|c|c|c|c|c|c|}
\hline \multirow{3}{*}{$\begin{array}{l}\text { Knowledge and } \\
\text { concerns relating to } \\
\text { Covid-19 }\end{array}$} & \multicolumn{8}{|l|}{ America } \\
\hline & \multicolumn{2}{|c|}{ Impact of event } & \multicolumn{2}{|l|}{ Stress } & \multicolumn{2}{|l|}{ Anxiety } & \multicolumn{2}{|l|}{ Depression } \\
\hline & $B$ & $t$ & $B$ & $t$ & $B$ & $t$ & $B$ & $t$ \\
\hline \multicolumn{9}{|l|}{ Mode of transmission } \\
\hline \multicolumn{9}{|l|}{ Droplets } \\
\hline Agree & 0.79 & $3.77 * * *$ & 0.48 & $2.88 * *$ & 1.42 & $5.90 * * *$ & 0.76 & $3.43 * *$ \\
\hline Disagree & \multicolumn{2}{|l|}{ Reference } & \multicolumn{2}{|l|}{ Reference } & \multicolumn{2}{|l|}{ Reference } & \multicolumn{2}{|l|}{ Reference } \\
\hline \multicolumn{9}{|c|}{ Contact with contaminated surfaces } \\
\hline Agree & 0.68 & $3.45^{* *}$ & 0.47 & $3.05 * *$ & 1.10 & $4.88 * * *$ & 0.71 & $3.44 * *$ \\
\hline Disagree & \multicolumn{2}{|l|}{ Reference } & \multicolumn{2}{|l|}{ Reference } & \multicolumn{2}{|l|}{ Reference } & \multicolumn{2}{|l|}{ Reference } \\
\hline \multicolumn{9}{|l|}{ Airborne } \\
\hline Agree & 0.38 & $3.46^{* *}$ & 0.19 & $2.21 *$ & 0.41 & $3.22 * *$ & 0.34 & $2.91 * *$ \\
\hline Disagree & \multicolumn{2}{|l|}{ Reference } & \multicolumn{2}{|l|}{ Reference } & Reference & & Reference & \\
\hline Degree of confidence & one's own & doctor to o & iagnose or & ecognize Co & & & & \\
\hline Very confident & -0.06 & -0.33 & -0.01 & -0.06 & -0.18 & -0.89 & -0.41 & -2.24 \\
\hline Somewhat confident & 0.02 & 0.13 & 0.05 & 0.37 & -0.02 & -0.10 & -0.28 & -1.66 \\
\hline Not very confident & 0.43 & 1.84 & 0.47 & $2.57 * \wedge$ & 0.45 & 1.68 & 0.09 & 0.35 \\
\hline Not confident & Reference & & Reference & & Reference & & Reference & \\
\hline Likelihood of contra & ng Covid-1 & 9 during th & e pandemic & & & & & \\
\hline Very possible & 1.67 & $5.14 * * * \prime$ & 1.20 & $4.64 * * * \prime$ & 1.48 & $3.86^{* * * \prime}$ & 1.71 & $4.97 * * * \prime$ \\
\hline Somewhat possible & 0.47 & 1.91 & 0.46 & 2.38 & 0.35 & 1.20 & 0.58 & 2.27 \\
\hline Not very possible & 0.15 & 0.62 & 0.25 & 1.27 & 0.21 & 0.72 & 0.40 & 1.56 \\
\hline Impossible & 0.36 & 1.44 & 0.36 & 1.81 & 0.22 & 0.76 & 0.40 & 1.53 \\
\hline Not sure & Reference & & Reference & & Reference & & Reference & \\
\hline Likelihood of surviv & if infected 1 & y Covid-19 & & & & & & \\
\hline Very possible & -0.44 & -1.74 & -0.36 & -1.82 & -0.28 & -0.97 & -0.22 & -0.82 \\
\hline Somewhat possible & -0.12 & -0.45 & -0.12 & -0.60 & 0.11 & 0.37 & 0.05 & 0.19 \\
\hline Not very possible & -0.12 & -0.40 & 0.05 & 0.19 & 0.45 & 1.29 & 0.52 & 1.66 \\
\hline Impossible & 0.29 & 0.89 & 0.02 & 0.09 & 0.75 & 1.98 & 0.64 & 1.87 \\
\hline Not sure & Reference & & Reference & & Reference & & Reference & \\
\hline Level of satisfaction & th the avail & able health & informatio & on Covid-1 & & & & \\
\hline Very satisfied & -0.13 & -0.53 & -0.13 & -0.67 & -0.15 & -0.53 & -0.17 & -0.67 \\
\hline Somewhat satisfied & -0.31 & -1.44 & -0.34 & -1.99 & -0.31 & -1.24 & -0.40 & -1.76 \\
\hline Not very satisfied & 0.08 & 0.32 & 0.28 & 1.48 & 0.43 & 1.51 & 0.33 & 1.31 \\
\hline Not satisfied & Reference & & Reference & & Reference & & Reference & \\
\hline Degree of worry abo & family mer & nbers being & diagnosed & with Covid-1 & & & & \\
\hline Very worried & -0.03 & -0.07 & -0.43 & -1.34 & -0.98 & -2.06 & -0.65 & -1.51 \\
\hline Somewhat worried & -0.50 & -1.22 & -0.64 & -1.98 & -1.11 & -2.32 & -0.90 & -2.09 \\
\hline Not very worried & -0.63 & -1.49 & -0.87 & $-2.58^{* \prime}$ & -1.55 & $-3.13 * * \prime$ & -1.14 & $-2.56^{* \prime}$ \\
\hline Not worried & -0.78 & -1.68 & -0.86 & -2.33 & -1.25 & -2.29 & -1.27 & $-2.58^{* \prime}$ \\
\hline No family members & Reference & & Reference & & Reference & & Reference & \\
\hline Did you feel discrim & ted agains & by other c & untries? & & & & & \\
\hline Yes & 1.08 & $5.73 * * *$ & 0.57 & $3.78 * * *$ & 1.11 & $5.06 * * *$ & 0.81 & $4.04 * * *$ \\
\hline No & Reference & & Reference & & Reference & & Reference & \\
\hline Mode of communica & n used to o & btain healt & informati & n on Covid- & & & & \\
\hline The Internet & -0.47 & $-3.09 * * \prime$ & -0.42 & $-3.52 * * * \prime$ & -0.69 & $-3.98 * * * \prime$ & -0.52 & $-3.33 * * \prime$ \\
\hline Television & -0.26 & -1.59 & -0.33 & $-2.56^{* \prime}$ & -0.53 & $-2.83^{* \prime}$ & -0.61 & $-3.59 * * * \prime$ \\
\hline Radio & -0.53 & -1.45 & -0.50 & -1.76 & -0.79 & -1.89 & -0.45 & -1.18 \\
\hline Family members & -0.12 & -0.29 & -0.06 & -0.20 & -0.33 & -0.70 & 0.21 & 0.49 \\
\hline Other modes & Reference & & Reference & & Reference & & Reference & \\
\hline
\end{tabular}


Table 3 (continued)

\begin{tabular}{|c|c|c|c|c|c|c|c|c|}
\hline \multirow{3}{*}{$\begin{array}{l}\text { Knowledge and } \\
\text { concerns relating to } \\
\text { Covid-19 }\end{array}$} & \multicolumn{8}{|l|}{ China } \\
\hline & \multicolumn{2}{|c|}{ Impact of event } & \multicolumn{2}{|l|}{ Stress } & \multicolumn{2}{|l|}{ Anxiety } & \multicolumn{2}{|l|}{ Depression } \\
\hline & $B$ & $t$ & $B$ & $t$ & $B$ & $t$ & $B$ & $t$ \\
\hline \multicolumn{9}{|c|}{ Mode of transmission } \\
\hline \multicolumn{9}{|l|}{ Droplets } \\
\hline Agree & -0.06 & -0.15 & -0.57 & -2.61 & -0.80 & $-2.26^{*}$ & -0.65 & $-2.24 *$ \\
\hline Disagree & Refere & ence & Reference & & Reference & & Reference & \\
\hline \multicolumn{9}{|c|}{ Contact with contaminated surfaces } \\
\hline Agree & -0.19 & -1.07 & -0.08 & -0.79 & 0.11 & 0.71 & 0.05 & 0.39 \\
\hline Disagree & Refere & ence & Reference & & Reference & & Reference & \\
\hline \multicolumn{9}{|l|}{ Airborne } \\
\hline Agree & 0.04 & 0.39 & 0.08 & 1.28 & 0.07 & 0.64 & 0.05 & 0.62 \\
\hline Disagree & Refere & ence & Reference & & Reference & & Reference & \\
\hline \multicolumn{9}{|c|}{ Degree of confidence in one's own doctor to diagnose or recognize Covid-19 } \\
\hline Very confident & 0.45 & 0.50 & -0.09 & -0.18 & -0.79 & -0.95 & -0.51 & -0.76 \\
\hline Somewhat confident & 0.75 & 0.83 & -0.02 & -0.03 & -0.70 & -0.85 & -0.39 & -0.57 \\
\hline Not very confident & 0.81 & 0.85 & 0.26 & 0.48 & -0.26 & -0.30 & -0.10 & -0.14 \\
\hline Not confident & Refere & ence & Reference & & Reference & & Reference & \\
\hline \multicolumn{9}{|c|}{ Likelihood of contracting Covid-19 during the pandemic } \\
\hline Very possible & -0.34 & $t-1.75$ & -0.06 & -0.57 & 0.07 & 0.38 & -0.06 & -0.42 \\
\hline Somewhat possible & -0.03 & -0.23 & -0.14 & -1.58 & -0.03 & -0.24 & -0.14 & -1.25 \\
\hline Not very possible & 0.05 & 0.34 & -0.15 & -1.77 & -0.08 & -0.57 & -0.15 & -1.36 \\
\hline Impossible & -0.12 & -0.65 & -0.19 & -1.90 & -0.15 & -0.92 & -0.31 & -2.33 \\
\hline Not sure & Refere & ence & Reference & & Reference & & Reference & \\
\hline \multicolumn{9}{|c|}{ Likelihood of survival if infected by Covid-19 } \\
\hline Very possible & -0.32 & -2.27 & -0.14 & -1.71 & -0.18 & -1.37 & -0.23 & -2.14 \\
\hline Somewhat possible & 0.02 & 0.12 & -0.09 & -1.18 & -0.09 & -0.83 & -0.19 & -2.00 \\
\hline Not very possible & -0.01 & -0.06 & 0.06 & 0.52 & 0.39 & 2.20 & 0.21 & 1.45 \\
\hline Impossible & -0.24 & -0.69 & 0.08 & 0.39 & 0.52 & 1.62 & 0.18 & 0.67 \\
\hline Not sure & Refere & ence & Reference & & Reference & & Reference & \\
\hline \multicolumn{9}{|c|}{ Level of satisfaction with the available health information on Covid-19 } \\
\hline Very satisfied & -0.86 & $5-2.99 * * \wedge$ & -0.67 & $-4.13 * * * \wedge$ & -0.87 & $-3.31 * * \wedge$ & -0.57 & $-2.64 * * \wedge$ \\
\hline Somewhat satisfied & -0.44 & -1.55 & -0.55 & $-3.46^{* * \wedge}$ & -0.78 & $-3.01 * * \wedge$ & -0.45 & -2.15 \\
\hline Not very satisfied & -0.68 & -2.02 & -0.38 & -2.00 & -0.59 & -1.93 & -0.16 & -0.62 \\
\hline Not satisfied & Refere & ence & Reference & & Reference & & Reference & \\
\hline \multicolumn{9}{|c|}{ Degree of worry about family members being diagnosed with Covid-19 } \\
\hline Very worried & -0.01 & -0.03 & -0.54 & $-2.57^{* \prime}$ & -0.41 & -1.21 & -0.78 & $-2.85^{* \prime}$ \\
\hline Somewhat worried & 0.12 & 0.34 & -0.64 & $-3.05 * * \prime$ & -0.40 & -1.21 & -0.81 & $-2.96^{* \prime}$ \\
\hline Not very worried & -0.29 & -0.79 & -0.75 & $-3.53 * * * \prime$ & -0.65 & -1.92 & -0.94 & $-3.39 * * \prime$ \\
\hline Not worried & -0.41 & -1.04 & -0.72 & $-3.14 * * \prime$ & -0.65 & -1.78 & -0.94 & $-3.15^{* * \prime}$ \\
\hline No family members & Refere & ence & Reference & & Reference & & Reference & \\
\hline \multicolumn{9}{|c|}{ Did you feel discriminated against by other countries? } \\
\hline Yes & 0.13 & 1.38 & 0.10 & 1.90 & 0.06 & 0.75 & 0.10 & 1.40 \\
\hline No & Refere & ence & Reference & & Reference & & Reference & \\
\hline \multicolumn{9}{|c|}{ Mode of communication used to obtain health information on Covid-19 } \\
\hline The Internet & -0.33 & -0.76 & -0.11 & -0.43 & -0.70 & -1.77 & -0.47 & -1.44 \\
\hline Television & -0.33 & -0.68 & -0.25 & -0.91 & -0.65 & -1.50 & -0.26 & -0.72 \\
\hline Radio & 0.11 & 0.13 & 2.11 & $4.30 * * * \prime$ & 1.89 & 2.42 & 2.33 & $3.64 * * * \prime$ \\
\hline Family members & 0.11 & 0.17 & -0.27 & -0.73 & -0.87 & -1.48 & -0.43 & -0.89 \\
\hline
\end{tabular}


Table 3 (continued)

\begin{tabular}{|c|c|c|c|c|c|c|c|}
\hline \multirow{3}{*}{$\begin{array}{l}\text { Knowledge and } \\
\text { concerns relating to } \\
\text { Covid-19 }\end{array}$} & \multicolumn{7}{|l|}{ China } \\
\hline & Impact of event & \multicolumn{2}{|c|}{ Stress } & \multicolumn{2}{|c|}{ Anxiety } & \multicolumn{2}{|c|}{ Depression } \\
\hline & $B$ & $B$ & $t$ & $B$ & $t$ & $B$ & $t$ \\
\hline Other modes & Reference & \multicolumn{2}{|c|}{ Reference } & \multicolumn{2}{|c|}{ Reference } & \multicolumn{2}{|c|}{ Reference } \\
\hline
\end{tabular}

${ }^{*} p<0.05 ; * * p<0.01$; *** $p<0.00$ (significance level was corrected by Bonferroni's method, $*^{\prime} p<0.0125, * *^{\prime} p<0.0025, * * * ' p<0.00025$; $* \wedge p<0.017, * * \wedge p<0.003, * * * \wedge<0.0003)$

\section{Precautionary health behaviors about COVID-19 and its association with psychological impact and adverse mental health status}

Overall, American and Chinese respondents demonstrated significantly different precautionary measures (see Supplementary Table 4 for detailed comparisons of engagement in precautionary measures between American and Chinese respondents). There were significantly more American respondents who endorsed regularly covering their mouths when coughing and sneezing, avoiding sharing cutlery, washing hands immediately after coughing/sneezing and washing hands with soap and water $(p<0.008)$. There were significantly more Chinese respondents who endorsed wearing a face mask $(p<0.008)$ and washing hands after touching contaminated objects $(p<0.017)$, and that they felt that there had been too much unnecessary worry about the COVID-19 pandemic $(p<0.008)$. There were significantly more Chinese respondents who spent $20-24 \mathrm{~h}$ per day for home confinement $(p<0.017)$.

Linear regression analysis showed that covering mouth when coughing and sneezing, avoidance of sharing cutlery (e.g., chopsticks) during meals, hand hygiene practices and wearing a face mask were significantly associated with lower scores on IES-R and DASS-21 sub-scales $(p<0.05)$ in Chinese respondents (see Table 4). It is important to note that wearing a face mask was associated with a higher IES-R score in American respondents $(p<0.001)$. American respondents who felt too much unnecessary worry had been made about the COVID-19 pandemic had significantly higher IES-R and DASS-21 subscale scores $(p<0.05)$. In contrast, Chinese respondents who felt that too much unnecessary worry had been made about the COVID-19 pandemic had significantly lower IES-R score $(p<0.05)$. American respondents who spent $0-10 \mathrm{~h}$ per day for home confinement had significantly lower IES-R scores $(p<0.05)$.

\section{Health information about COVID-19 and its association with psychological impact and adverse mental health status}

American and Chinese respondents were also significantly different in desired information about COVID-19. There were significantly more Chinese respondents who indicated a need for information on the symptoms related to COVID19 , prevention methods, treatment methods, regular information updates, local outbreaks, more personalized information, the effectiveness of drugs and vaccines, number of infected by geographical locations, travel advice and transmission methods as compared to American $(p<0.001)$ (See Supplementary Table 5).

Positive responses on items related to desire for information including symptoms of COVID-19, prevention advice, treatment methods, need for regular information updates, more knowledge for local transmission, more personalized information related to COVID-19, need to know the effectiveness of drugs and vaccines, need to know the number of people infected and geographical location, travel advice, need to understand transmission methods of COVID-19 and need to know other countries' response to COVID-19 were significantly associated with higher IES-R scores or one of the DASS-21 subscales for American respondents. $(p<0.05)$ (see Table 5). Items related to the need for additional knowledge and for local transmission, need for travel advice, and need to know other countries' response to COVID-19 were significantly associated with lower DASS-21 subscale scores in both countries $(p<0.05)$.

\section{Discussion}

To our best knowledge, this is the first study that compared the physical and mental health between citizens from the U.S. and China. Our results supported the hypothesis and there were different characteristics between American and Chinese respondents in mental and physical health parameters, health behaviors, precautionary measures and knowledge of COVID-19. In July 2020, the total number of COVID-19 cases and death cases in the U.S. were much greater than those in China (JHU, 2020). Likely as a result, a significantly higher proportion of American respondents reported physical symptoms resembling COVID-19, doctor consultation and testing of COVID-19, contact history with COVID-19 and worried about their family members contracting COVID19. Although more American respondents reportedly utilized 
Table 4 Comparison of the association between precautionary measures related to COVID-19 and the psychological impact as well as adverse mental health status between American and Chinese participants (U.S. $\mathrm{n}=584$, China $\mathrm{n}=861$, Total $\mathrm{N}=1445$ )

\begin{tabular}{|c|c|c|c|c|c|c|c|c|}
\hline \multirow{3}{*}{$\begin{array}{l}\text { Precau- } \\
\text { tionary } \\
\text { meas- } \\
\text { ures }\end{array}$} & \multicolumn{8}{|l|}{ The US } \\
\hline & \multicolumn{2}{|c|}{ Impact of event } & \multicolumn{2}{|l|}{ Stress } & \multicolumn{2}{|l|}{ Anxiety } & \multicolumn{2}{|l|}{ Depression } \\
\hline & $B$ & $t$ & $B$ & $t$ & $B$ & $t$ & $B$ & $t$ \\
\hline \multicolumn{9}{|c|}{ Covers mouth when coughing or sneezing } \\
\hline Always & $<0.001$ & $<0.001$ & -0.27 & -0.95 & -0.58 & -1.43 & -0.10 & -0.26 \\
\hline Often & -0.09 & -0.24 & -0.08 & -0.26 & -0.42 & -1.00 & 0.16 & 0.40 \\
\hline $\begin{array}{l}\text { Some- } \\
\text { times }\end{array}$ & 1.00 & $2.50 *$ & 0.28 & 0.87 & 0.69 & 1.51 & 0.81 & 1.92 \\
\hline never & Reference & & Referenc & & Reference & & Reference & \\
\hline \multicolumn{9}{|c|}{ Avoidance of sharing cutlery during meals } \\
\hline Always & 0.07 & 0.26 & -0.10 & -0.47 & -0.41 & -1.36 & -0.39 & -1.43 \\
\hline Often & 0.32 & 1.11 & -0.04 & -0.17 & -0.13 & -0.39 & -0.28 & -0.92 \\
\hline $\begin{array}{l}\text { Some- } \\
\text { times }\end{array}$ & 0.77 & $2.45^{* \prime}$ & 0.41 & 1.64 & 0.82 & $2.27 *$ & 0.39 & 1.17 \\
\hline Never & Reference & & Referenc & & Reference & & Reference & \\
\hline \multicolumn{9}{|c|}{ Washing your hands using soap or hand sanitizer } \\
\hline Always & 0.27 & 0.49 & -0.19 & -0.44 & -0.67 & -1.05 & -0.41 & -0.72 \\
\hline Often & 0.25 & 0.44 & -0.05 & -0.11 & -0.57 & -0.87 & -0.24 & -0.41 \\
\hline $\begin{array}{l}\text { Some- } \\
\text { times }\end{array}$ & 0.52 & 0.90 & -0.12 & -0.26 & -0.21 & -0.31 & -0.13 & -0.21 \\
\hline Never & Reference & & Referenc & & Reference & & Reference & \\
\hline \multicolumn{9}{|c|}{ Washing hands immediately after coughing, sneezing or rubbing your nose } \\
\hline Always & 0.14 & 0.49 & -0.33 & -1.45 & -0.48 & -1.43 & -0.31 & -1.02 \\
\hline Often & -0.002 & -0.01 & -0.37 & -1.54 & -0.60 & -1.73 & -0.19 & -0.62 \\
\hline $\begin{array}{l}\text { Some- } \\
\text { times }\end{array}$ & 0.07 & 0.22 & -0.26 & -1.06 & -0.42 & -1.18 & 0.01 & 0.03 \\
\hline Never & Reference & & Referenc & & Reference & & Reference & \\
\hline \multicolumn{9}{|c|}{ Wearing a face mask regardless of the presence or absence of symptoms } \\
\hline Always & 0.52 & $3.47 * * \prime$ & 0.07 & 0.62 & 0.32 & 1.84 & 0.06 & 0.40 \\
\hline Often & 0.24 & 1.34 & -0.09 & -0.60 & 0.07 & 0.32 & -0.06 & -0.29 \\
\hline $\begin{array}{l}\text { Some- } \\
\text { times }\end{array}$ & 0.28 & 1.60 & -0.02 & -0.16 & 0.36 & 1.79 & 0.16 & 0.88 \\
\hline Never & Reference & & Referenc & & Reference & & Reference & \\
\hline \multicolumn{9}{|c|}{ Washing hands after coming into contact with contaminated surfaces } \\
\hline Always & 0.10 & 0.25 & -0.28 & -0.85 & -0.17 & -0.36 & -0.23 & -0.55 \\
\hline Often & 0.001 & 0.003 & -0.30 & -0.89 & -0.12 & -0.25 & -0.16 & -0.36 \\
\hline $\begin{array}{l}\text { Some- } \\
\text { times }\end{array}$ & 0.32 & 0.72 & -0.04 & -0.12 & 0.44 & 0.86 & 0.33 & 0.71 \\
\hline Never & Reference & & Referenc & & Reference & & Reference & \\
\hline \multicolumn{9}{|c|}{ People are too worried about Covid-19 } \\
\hline Always & 0.43 & 2.09 & 0.39 & $2.39 * \prime$ & 0.54 & 2.27 & 0.32 & 1.47 \\
\hline Often & 0.33 & 1.83 & 0.25 & 1.73 & 0.67 & $3.21 * * \prime$ & 0.48 & $2.53^{* \prime}$ \\
\hline $\begin{array}{l}\text { Some- } \\
\text { times }\end{array}$ & 0.20 & 1.79 & -0.02 & -0.19 & 0.25 & 1.94 & 0.02 & 0.16 \\
\hline Never & Reference & & Referenc & & Reference & & Reference & \\
\hline \multicolumn{9}{|c|}{ Time spent at home (hours) } \\
\hline$[0-10]$ & -0.26 & $-2.26^{* \wedge}$ & -0.06 & -0.61 & -0.29 & -2.16 & -0.19 & -1.56 \\
\hline (10-20] & 0.04 & 0.29 & 0.09 & 0.85 & -0.004 & -0.03 & -0.08 & -0.54 \\
\hline$(20-24]$ & Reference & & Referenc & & Reference & & Reference & \\
\hline
\end{tabular}


Table 4 (continued)

\begin{tabular}{|c|c|c|c|c|c|c|c|c|}
\hline \multirow{3}{*}{$\begin{array}{l}\text { Precau- } \\
\text { tionary } \\
\text { meas- } \\
\text { ures }\end{array}$} & \multicolumn{8}{|l|}{ China } \\
\hline & \multicolumn{2}{|c|}{ Impact of event } & \multicolumn{2}{|l|}{ Stress } & \multicolumn{2}{|l|}{ Anxiety } & \multicolumn{2}{|l|}{ Depression } \\
\hline & $B$ & $t$ & $B$ & $t$ & $B$ & $t$ & $B$ & $t$ \\
\hline \multicolumn{9}{|c|}{ Covers mouth when coughing or sneezing } \\
\hline Always & -0.37 & $-2.52^{* \prime}$ & -0.35 & $-4.11 * * * \prime$ & -0.51 & $-3.81 * * * \prime$ & -0.42 & $-3.84 * * * \prime$ \\
\hline Often & -0.10 & -0.60 & -0.37 & $-3.93 * * * \prime$ & -0.47 & $-3.14 * * \prime$ & -0.31 & $-2.48 * \prime$ \\
\hline $\begin{array}{l}\text { Some- } \\
\text { times }\end{array}$ & 0.03 & 0.12 & -0.24 & -1.98 & -0.47 & $-2.42^{* \prime}$ & -0.26 & -1.61 \\
\hline never & Reference & & Referenc & & Reference & & Reference & \\
\hline \multicolumn{9}{|c|}{ Avoidance of sharing cutlery during meals } \\
\hline Always & -0.48 & $-4.28 * * * \prime$ & -0.21 & $-3.09 * * \prime$ & -0.29 & $-2.72 * \prime$ & -0.28 & $-3.18 * * \prime$ \\
\hline Often & -0.01 & -0.07 & -0.20 & $-2.44 * \prime$ & -0.18 & -1.37 & -0.19 & -1.78 \\
\hline $\begin{array}{l}\text { Some- } \\
\text { times }\end{array}$ & 0.05 & 0.36 & -0.17 & -1.99 & -0.17 & -1.29 & -0.24 & $-2.20 * \prime$ \\
\hline Never & Reference & & Referenc & & Reference & & Reference & \\
\hline \multicolumn{9}{|c|}{ Washing your hands using soap or hand sanitizer } \\
\hline Always & -0.39 & $-2.30^{* \prime}$ & -0.24 & $-2.44^{* \prime}$ & -0.25 & -1.60 & -0.34 & $-2.66^{* \prime}$ \\
\hline Often & 0.16 & 0.88 & -0.15 & -1.45 & -0.03 & -0.16 & -0.21 & -1.51 \\
\hline $\begin{array}{l}\text { Some- } \\
\text { times }\end{array}$ & -0.10 & -0.49 & -0.27 & -2.24 & -0.06 & -0.29 & -0.34 & -2.13 \\
\hline Never & Reference & & Referenc & & Reference & & Reference & \\
\hline \multicolumn{9}{|c|}{ Washing hands immediately after coughing, sneezing or rubbing your nose } \\
\hline Always & -0.55 & $-4.44 * * * \prime$ & -0.22 & $-2.96^{* * \prime}$ & -0.27 & -2.34 & -0.26 & $-2.76^{* \prime}$ \\
\hline Often & 0.01 & 0.09 & -0.11 & -1.34 & -0.08 & -0.63 & -0.15 & -1.40 \\
\hline $\begin{array}{l}\text { Some- } \\
\text { times }\end{array}$ & -0.06 & -0.37 & -0.12 & -1.28 & -0.13 & -0.93 & -0.14 & -1.17 \\
\hline Never & Reference & & Referenc & & Reference & & Reference & \\
\hline \multicolumn{9}{|c|}{ Wearing a face mask regardless of the presence or absence of symptoms } \\
\hline Always & -0.70 & $-2.70^{* \prime}$ & -0.34 & -2.20 & -0.54 & -2.23 & -0.46 & -2.32 \\
\hline Often & -0.30 & -1.10 & -0.27 & -1.68 & -0.34 & -1.34 & -0.26 & -1.24 \\
\hline $\begin{array}{l}\text { Some- } \\
\text { times }\end{array}$ & -0.36 & -1.13 & -0.27 & -1.46 & -0.37 & -1.27 & -0.32 & -1.30 \\
\hline Never & Reference & & Referenc & & Reference & & Reference & \\
\hline \multicolumn{9}{|c|}{ Washing hands after coming into contact with contaminated surfaces } \\
\hline Always & -0.39 & -1.34 & -0.53 & $-3.12 * * \prime$ & -0.81 & $-3.04 * * \prime$ & -0.80 & $-3.66 * * * \prime$ \\
\hline Often & 0.003 & 0.01 & -0.48 & $-2.71 * \prime$ & -0.60 & -2.15 & -0.62 & $-2.73 * * \prime$ \\
\hline $\begin{array}{l}\text { Some- } \\
\text { times }\end{array}$ & 0.22 & 0.59 & -0.38 & -1.74 & -0.43 & -1.25 & -0.59 & -2.07 \\
\hline Never & Reference & & Referenc & & Reference & & Reference & \\
\hline \multicolumn{9}{|c|}{ People are too worried about Covid-19 } \\
\hline Always & -0.48 & $-3.64 * * * \prime$ & 0.09 & 1.15 & 0.07 & 0.60 & 0.09 & 0.91 \\
\hline Often & -0.02 & -0.12 & 0.18 & 1.94 & 0.36 & $2.48^{* \prime}$ & 0.29 & $2.46^{* \prime}$ \\
\hline $\begin{array}{l}\text { Some- } \\
\text { times }\end{array}$ & -0.05 & -0.52 & -0.05 & -0.91 & -0.02 & -0.18 & 0.03 & 0.41 \\
\hline Never & Reference & & Referenc & & Reference & & Reference & \\
\hline \multicolumn{9}{|c|}{ Time spent at home (hours) } \\
\hline$[0-10]$ & -0.41 & -1.41 & 0.16 & 0.96 & 0.26 & 0.96 & 0.35 & 1.58 \\
\hline (10-20] & 0.22 & 1.45 & 0.10 & 1.13 & 0.17 & 1.18 & 0.01 & 0.12 \\
\hline (20-24] & Reference & & Referenc & & Reference & & Reference & \\
\hline
\end{tabular}

${ }^{*} p<0.05 ; * * p<0.01 ; * * * p<0.001$ (significance level was corrected by Bonferroni's method, ${ }^{*} p<0.017, * * \prime p<0.003, * * * \prime p<0.0003$; $\left.{ }^{* \wedge} p<0.025\right)$ 
Table 5 Comparison of the association between information needs about COVID-19 between American and Chinese participants and the psychological impact as well as adverse mental health status between
American and Chinese participants (U.S. $\mathrm{n}=584$, China $\mathrm{n}=861$, Total $\mathrm{N}=1445$ )

\begin{tabular}{|c|c|c|c|c|c|c|c|c|}
\hline \multirow{3}{*}{$\begin{array}{l}\text { Information } \\
\text { needs }\end{array}$} & \multicolumn{8}{|l|}{ The US } \\
\hline & \multicolumn{2}{|c|}{ Impact of event } & \multicolumn{2}{|l|}{ Stress } & \multicolumn{2}{|l|}{ Anxiety } & \multicolumn{2}{|l|}{ Depression } \\
\hline & $B$ & $t$ & $B$ & $t$ & $B$ & $t$ & $B$ & $t$ \\
\hline \multicolumn{9}{|c|}{ Symptoms related to Covid-19 } \\
\hline Yes & 0.56 & $5.40 * * *$ & 0.34 & $4.11 * * *$ & 0.70 & $5.92 * * *$ & 0.46 & $4.27 * * *$ \\
\hline No & Reference & & Reference & & Reference & & Reference & \\
\hline \multicolumn{9}{|c|}{ Prevention advice } \\
\hline Yes & 0.51 & $4.84 * * *$ & 0.30 & $3.59 * * *$ & 0.60 & $4.98 * * *$ & 0.32 & $2.92 * *$ \\
\hline No & Reference & & Reference & & Reference & & Reference & \\
\hline \multicolumn{9}{|c|}{ Treatment methods } \\
\hline Yes & 0.46 & $4.50 * * *$ & 0.19 & $2.39 *$ & 0.45 & $3.79 * * *$ & 0.33 & $3.08 * *$ \\
\hline No & Reference & & Reference & & Reference & & Reference & \\
\hline \multicolumn{9}{|c|}{ Need for regular information updates } \\
\hline Yes & 0.28 & $2.74 * *$ & 0.14 & 1.75 & 0.31 & $2.70^{* *}$ & 0.22 & $2.04 *$ \\
\hline No & Reference & & Reference & & Reference & & Reference & \\
\hline \multicolumn{9}{|c|}{ Need for knowledge on local transmissions } \\
\hline Yes & 0.18 & 1.79 & 0.07 & 0.81 & 0.28 & $2.42 *$ & 0.16 & 1.49 \\
\hline No & Reference & & Reference & & Reference & & Reference & \\
\hline \multicolumn{9}{|c|}{ Need for more personalized information, such as advice for those with pre-existing medical conditions } \\
\hline Yes & 0.42 & $4.07 * * *$ & 0.21 & $2.51 *$ & 0.49 & $4.06 * * *$ & 0.31 & $2.83^{* *}$ \\
\hline No & Reference & & Reference & & Reference & & Reference & \\
\hline \multicolumn{9}{|c|}{ Need to know the effectiveness of drugs and vaccines } \\
\hline Yes & 0.23 & $2.25 *$ & 0.07 & 0.87 & 0.27 & $2.29 *$ & 0.17 & 1.58 \\
\hline No & Reference & & Reference & & Reference & & Reference & \\
\hline \multicolumn{9}{|c|}{ Need to know the number of people infected, and geographical location } \\
\hline Yes & 0.29 & $2.83 * *$ & 0.15 & 1.88 & 0.33 & $2.85 * *$ & 0.15 & 1.37 \\
\hline No & Reference & & Reference & & Reference & & Reference & \\
\hline \multicolumn{9}{|c|}{ Need for travel advice } \\
\hline Yes & 0.41 & $3.85 * * *$ & 0.12 & 1.38 & 0.40 & $3.25 * *$ & 0.25 & $2.25^{*}$ \\
\hline No & Reference & & Reference & & Reference & & Reference & \\
\hline \multicolumn{9}{|c|}{ Need to understand transmission methods of Covid-19 } \\
\hline Yes & 0.54 & $5.24 * * *$ & 0.27 & $3.27 * *$ & 0.58 & $4.85 * * *$ & 0.33 & $2.99 * *$ \\
\hline No & Reference & & Reference & & Reference & & Reference & \\
\hline \multicolumn{9}{|c|}{ Need to know other countries' response to Covid-19 } \\
\hline Yes & 0.36 & $3.51 * * *$ & 0.21 & $2.59 *$ & 0.44 & $3.73 * * *$ & 0.27 & $2.50^{*}$ \\
\hline No & Reference & & Reference & & Reference & & Reference & \\
\hline \multirow{3}{*}{$\begin{array}{l}\text { Information } \\
\text { needs }\end{array}$} & China & & & & & & & \\
\hline & Impact of e & & Stress & & Anxiety & & Depression & \\
\hline & $B$ & $t$ & $B$ & $t$ & $B$ & $t$ & $B$ & $t$ \\
\hline \multicolumn{9}{|c|}{ Symptoms related to Covid-19 } \\
\hline Yes & 0.23 & 1.83 & 0.03 & 0.43 & 0.11 & 0.96 & 0.01 & 0.10 \\
\hline No & Reference & & Reference & & Reference & & Reference & \\
\hline \multicolumn{9}{|c|}{ Prevention advice } \\
\hline Yes & 0.28 & 1.91 & -0.10 & -1.17 & -0.15 & -1.12 & -0.15 & -1.37 \\
\hline No & Reference & & Reference & & Reference & & Reference & \\
\hline \multicolumn{9}{|c|}{ Treatment methods } \\
\hline Yes & 0.24 & $2.33^{*}$ & 0.09 & 1.54 & 0.21 & $2.24 *$ & 0.10 & 1.23 \\
\hline
\end{tabular}


Table 5 (continued)

\begin{tabular}{|c|c|c|c|c|c|c|c|c|}
\hline \multirow{3}{*}{$\begin{array}{l}\text { Information } \\
\text { needs }\end{array}$} & \multicolumn{8}{|l|}{ China } \\
\hline & \multicolumn{2}{|c|}{ Impact of event } & \multicolumn{2}{|l|}{ Stress } & \multicolumn{2}{|l|}{ Anxiety } & \multicolumn{2}{|l|}{ Depression } \\
\hline & $B$ & $t$ & $B$ & $t$ & $B$ & $t$ & $B$ & $t$ \\
\hline No & Reference & & Reference & & Reference & & Reference & \\
\hline \multicolumn{9}{|c|}{ Need for regular information updates } \\
\hline Yes & 0.47 & $2.29 *$ & -0.14 & -1.18 & -0.12 & -0.63 & -0.21 & -1.32 \\
\hline No & Reference & & Reference & & Reference & & Reference & \\
\hline \multicolumn{9}{|c|}{ Need for knowledge on local transmissions } \\
\hline Yes & 0.15 & 0.66 & -0.33 & $-2.60^{*}$ & -0.30 & -1.49 & -0.37 & $-2.20^{*}$ \\
\hline No & Reference & & Reference & & Reference & & Reference & \\
\hline \multicolumn{9}{|c|}{ Need for more personalized information, such as advice for those with pre-existing medical conditions } \\
\hline Yes & 0.07 & 0.48 & -0.13 & -1.56 & -0.16 & -1.24 & -0.19 & -1.81 \\
\hline No & Reference & & Reference & & Reference & & Reference & \\
\hline \multicolumn{9}{|c|}{ Need to know the effectiveness of drugs and vaccines } \\
\hline Yes & 0.17 & 1.01 & -0.17 & -1.68 & -0.08 & -0.53 & -0.22 & -1.71 \\
\hline No & Reference & & Reference & & Reference & & Reference & \\
\hline \multicolumn{9}{|c|}{ Need to know the number of people infected, and geographical location } \\
\hline Yes & 0.17 & 1.01 & -0.08 & -0.81 & -0.13 & -0.86 & -0.17 & -1.31 \\
\hline No & Reference & & Reference & & Reference & & Reference & \\
\hline \multicolumn{9}{|c|}{ Need for travel advice } \\
\hline Yes & 0.14 & 1.04 & -0.16 & $-2.06^{*}$ & -0.06 & -0.51 & -0.25 & $-2.45^{*}$ \\
\hline No & Reference & & Reference & & Reference & & Reference & \\
\hline \multicolumn{9}{|c|}{ Need to understand transmission methods of Covid-19 } \\
\hline Yes & 0.53 & $2.94 * *$ & -0.11 & -1.07 & -0.17 & -1.03 & -0.15 & -1.12 \\
\hline No & Reference & & Reference & & Reference & & Reference & \\
\hline \multicolumn{9}{|c|}{ Need to know other countries' response to Covid-19 } \\
\hline Yes & 0.32 & $3.45^{* *}$ & -0.02 & -0.33 & -0.13 & -1.54 & -0.16 & $-2.33^{*}$ \\
\hline No & Reference & & Reference & & Reference & & Reference & \\
\hline
\end{tabular}

${ }^{*} p<0.05 ; * * p<0.01 ; * * * p<0.01$

medical services recently, more Chinese respondents were covered by medical insurances than their U.S. counterparts. Previous surveys found that health insurance coverage for China and the U.S. were $95 \%$ and $89 \%$ respectively (LeDeu et al., 2012; CBO, 2019), but U.S. unemployment during the pandemic may rapidly shift those numbers. Despite a higher number of COVID-19 cases in the U.S., a significantly lower proportion of American respondents used face masks as a precaution and the use of face masks was associated with higher IES-R scores. For health information and COVID19 knowledge, American respondents were more dissatisfied with health information and spent more time monitoring health information. For American respondents, it appeared that seeking additional health information was associated with adverse mental health. In contrast, for Chinese respondents, additional health information had protective effects on some of the mental health parameters.

For mental health parameters, American respondents reported significantly higher levels of depressive and stress symptoms than Chinese respondents, but the Chinese respondents reported higher levels of PTSD symptoms and perceived discrimination by other countries during the COVID-19 pandemic. The statistical significance of these findings are tempered by the small absolute differences in the distress scores of the DASS-21 between countries and should be interpreted cautiously (Ronk et al., 2013). Protective factors against adverse mental health for the American respondents included student status, being a parent living with a child older than 16 years of age and spending less time in home confinement. Protective factors against adverse mental health in China included higher levels of confidence in their doctors, wearing face masks, obtaining information on travel advice, and obtaining information on other countries' responses during the COVID-19 pandemic. For both countries, risk factors for adverse mental health included the presence of physical symptoms resembling COVID-19 infection (e.g., headache, dizziness, cough, difficulty in breathing, gastrointestinal symptoms, persistent fever, chills and coryza) and recent quarantine. Precautionary health behaviors (e.g., covering mouth when coughing/sneezing, certain hand hygiene measures, wearing a face 
mask) were protective factors for mental health for Chinese respondents but not for US participants. These results suggest that both American and Chinese respondents appeared to be monitoring and reactive to physical symptoms that are known to be associated with COVID-19, but that are also commonly experienced due to benign conditions. This potential overlap of symptoms and the resulting attentional bias likely contribute to the psychological burden of the current pandemic.

The most important implication of the present study is to understand the differences between the two largest economies and better prepare for the next pandemic. The higher levels of stress and depression and lower confidence of American respondents in their medical services were likely due to the rising number of infected healthcare workers in the U.S. (Cohen, 2020), shortage of personal protective equipment (Schlanger, 2020), and the vulnerability of losing personal health insurance tied to their employment. Interestingly, we also found that the levels of anxiety in American respondents were not significantly higher than Chinese respondents. A recent study found that the implementation of stay-at-home orders in the U.S. are associated with a significant flattening of the curve associated with anxiety during the COVID-19 pandemic because known governmental action prevented further fears and unrest (Jacobson et al., 2020), Nevertheless, Chinese respondents reported higher level of IES-R scores or PTSD symptoms, likely due to stricter government mandated prevention and control measures as compared to the U.S. (CDC, 2020). The impact of COVID-19 to the U.S. and China mental health service has been disruptive in various settings including outpatient clinics, emergency departments, inpatient wards, and community services (Bojdani et al., 2020; Hao et al., 2020).

Many research studies have supported the benefits of face masks in blocking virus transmission in aerosols but were opposed by erroneous judgment (Ma et al., 2020). Wearing a face mask can impede the spread of the virus from asymptomatic COVID-19 patients (Zhai, 2020). Nevertheless, this study found that American respondents were significantly less likely to wear face masks as compared to Chinese. Besides potential benefits on physical health, wearing face masks could offer psychological benefits as it was associated with a lower prevalence of depression in Chinese respondents. In contrast, frequency of mask-wearing was associated with higher IES-R scores in American respondents. The American public may hold ambivalent views towards face masks due to mask-wearing being perceived as a symbol of sickness and concealment of identity.

The COVID-19 pandemic has prompted the U.S. and China to work together for the well-being of their citizens. As physical symptoms resembling COVID-19 infection (e.g., headache, dizziness, cough, difficulty in breathing, gastrointestinal symptoms) were associated with adverse mental health in both countries, the limitations of testing for coronavirus could worsen stress and depression. There is an urgent need to develop accurate and rapid diagnostic tests in family doctors' clinics. Nevertheless, there is a need to improve quality of health information related COVID-19 (Tran et al., 2020a) as American respondents spent more time on the Internet to obtain health information. A study analyzed trends of information inquiry related to COVID19 in New York State; most inquiries were related to gather information on the coronavirus, followed by people requesting information about symptoms, prevention and testing for coronavirus (Lieberman-Cribbin et al., 2020). Further research is required to understand the perceptions of the American public towards health information related to COVID-19 and explore the underlying reasons between adverse mental health and health information (e.g., symptoms, prevention methods, transmission methods).

\section{Strengths and limitations}

The main strength of this study was that we performed indepth analysis and studied the relationship between psychological outcomes and other variables related to COVID-19 in the populations of the two largest economies in the World. However, there are several limitations to be considered when interpreting the results. One major limitation was that the American and Chinese respondents were not matched by age, marital status and occupation. The differences between American and Chinese respondents could be due to intrinsic differences in age and other demographic factors. However, it is also notable that a majority of both Chinese and American respondents were college-educated, likely representing an unintentional sampling bias toward individuals who have greater access to information regarding the pandemic and resources to cope with psychologic distress related to the pandemic. Another limitation is that the electronic survey data did not include specific geographic information (e.g., specific city, state) that may have been helpful in assessing differences in rural and urban response to the pandemic. In addition, the impact of the relative duration of the crisis was not addressed in this study. The results of this study primarily address the first 3-6 months of the pandemic response but long-term effects are not addressed here. The second limitation was the potential risk of sampling bias. This bias could be due to the online administration of questionnaires and the majority of respondents from both countries were respondents with a good educational background with Internet access. We could not reach out to potential respondents without Internet access or staying in rural areas (Tran et al., 2020c). Further, we did not include measures of cultural factors that might further explain why differences may be present between the two samples. Both countries have significant regional and cultural traditions that likely provide 
some variability in the responses. Future research may want to identify different areas of each country more carefully for closer comparisons with matching strategies.

\section{Conclusion}

The current study highlighted potential differences in the impact of the COVID-19 pandemic in American and Chinese respondents. Likely due to a higher number of COVID19 cases per capita in the U.S., American respondents reported more physical symptoms, contact history, higher perceived likelihood of contracting COVID-19, frequent use of medical services and less confidence in medical services. American respondents also reported higher levels of depression and stress, while the Chinese respondents reported higher levels of acute traumatic stress symptoms. Chinese respondents encountered more discrimination by other countries. There were likely cultural differences regarding the use of face masks and mental health responses to health information related to COVID-19. Our findings suggest that the differences and similarities of the U.S. and China in response to the COVID-19 may need to be considered as we attempt various public health policies in each country to safeguard global physical and mental health.

Acknowledgements This study has the following funding sources: National University of Singapore iHeathtech Other Operating Expenses (R-722-000-004-731). The corresponding author has full access to all the data in the study and had final responsibility for the decision to submit for publication.

Author contributions Concept and design: CW, CT, SS, RP, RH. Acquisition, analysis, and interpretation of data: CW, CT, SS, RP, LX, TQ, XW, YT. Drafting of the manuscript: CW, CT, SS, NAC, CH, RH. Critical revision of the manuscript: CT, SS, CH. Statistical analysis: CW, CT, SS, RP, LX, FC, XW, YT.

\section{Declarations}

Conflict of interest The authors declare that they have no known competing for financial interests or personal relationships that could have appeared to influence the work reported in this paper.

Human and animal rights and Informed consent All procedures followed were in accordance with ethical standards of the responsible committee on human experimentation (institutional and national) and with the Helsinki Declaration of 1975, as revised in 2000. Informed consent was obtained from all patients for being included in the study.

\section{References}

Bojdani, E., Rajagopalan, A., Chen, A., et al. (2020). COVID-19 pandemic: Impact on psychiatric care in the United States, a review. Psychiatry Research, 289, 1-6.
Center for Disease Control (CDC) China. (2020). Prevention and control measure of COVID-19 in China. Retrieved June 20, 2020, from https://www.who.int/docs/default-source/wpro--documents/ countries/china/covid-19-briefing-nhc/11-slides-of-dr-li-zhongjie. pdf?sfvrsn=a1dedfe7_2.

Chew, N. W. S., Lee, G. K. H., Tan, B. Y. Q., et al. (2020). A multination, multicentre study on the psychological outcomes and associated physical symptoms amongst healthcare workers during COVID-19 outbreak. Brain, Behavior, and Immunity. https://doi. org/10.1016/j.bbi.2020.04.049

Cohen, E. (2020). CDC estimates more than 9,200 healthcare workers have been infected with COVID-19. Retrieved June 20, 2020, from https://edition.cnn.com/2020/04/15/health/coronavirus-9200health-workers-infected/index.html.

Congressional Budget Office (CBO). (2019). Federal subsidies for health insurance coverage for people under age 65: 2019 to 2029. Retrieved June 27, 2020, from https://www.cbo.gov/publication/ 55085.

Creamer, M., Bell, R., \& Failla, S. (2003). Psychometric propertics of the impact of event scale-Revised. Behaviour Research and Therapy, 41, 1489-1496.

Hao, F. T. W., Jang, L., Zhang, L., et al. (2020). Do psychiatric patients experience more psychiatric symptoms during COVID-19 pandemic and lockdown? A case-control study with service and research implications for immuno psychiatry. Brain, Behavior, and Immunity, 87, 100-106.

Ho, C. S., Chee, C. Y., \& Ho, R. C. (2020a). Mental health strategies to combat the psychological impact of COVID-19 beyond paranoia and panic. Annals of the Academy of Medicine Singapore, 49, 1-3.

Ho, C. S. H., Tan, E. L. Y., Ho, R. C. M., \& Chiu, M. Y. L. (2020b). Relationship of anxiety and depression with respiratory symptoms: Comparison between depressed and non-depressed smokers in Singapore. International Journal of Environmental Research and Public Health, 16, 163-177.

Ho, R. C., Zhang, M. W., Ho, C. S., et al. (2014). Impact of 2013 south Asian haze crisis: Study of physical and psychological symptoms and perceived dangerousness of pollution level. BMC Psychiatry, $24,1-9$.

Hosey, M. M., Bienvenu, O. J., Dinglas, V. D., et al. (2019). The IES-R remains a core outcome measrue for PTSD in critical illness survivorship research. Critical Care, 23, 362.

Jacobson, N. C., Lekkas, D., Price, G., et al. (2020). Flattening the mental health curve: COVID-19 stay-at-home orders are associated with alterations in mental health search behavior in United States. Journal of Medical Internet Research Mental Health, 7, e19347.

John Hopkins University. (2020). COVID-19 dashboard by the Center for Systems Science and Engineering (CSSE) at John Hopkins University (JHU). Retrieved July 6, 2020, from https:// coronavirus.jhu.edu/map.html.

Le, T. A., Le, M. Q. T., Dang, A. D., et al. (2019). Multi-level predictors of psychological problems among methadone maintenance treatment patients in different types of setting in Vietnam. Substance Abuse: Treatment, Prevention, and Policy, 14, 39.

LeDeu, T., Le, M. Q. T., Dang, A. D., et al. (2012). Healthcare in China: Entering 'unchartered waters.” Retrieved June 27, 2020, from https://www.mckinsey.com/industries/healthcare-systemsand-services/our-insights/health-care-in-china-entering-uncha rted-waters\#.

Lee, S. M., Kang, W. S., Cho, A. R., Kim, T., \& Park, J. K. (2018). Psychological impact of the 2015 MERS outbreak on hospital workers and quarantined hemodialysis patients. Comprehensive Psychiatry, 87, 123-127.

Lieberman-Cribbin, W., Alpert, N., Gonzalez, A., Schwartz, R. M., \& Taioli, E. (2020). Three months of information trends in 
COVID-19 across New York City. Journal of Public Health, $42,448-450$.

Liu, C. H., Zhang, E., Wong, G. T. F., Hyun, S., \& Hahm, H. C. (2020). Factors associated with depression, anxiety, and PTSD symptomology during the COVID-19 pandemic: Clinical implication for US young adult mental health. Psychiatry Research, 290, 1-7.

Ma, Q. X., Shan, J., Zhan, H. L., et al. (2020). Potential utilities of mask-wearing and instand hand hygeine for fighting SARSCoV-2. Journal of Medical Virology, 92, 1567-1571.

Mahase, E. (2020). COVID-19: WHO declares pandemic because of "alarming levels" of spread, severity, and inaction. British Medical Journal, 368, m1036.

Norton, P. J. (2009). Depression anxiety and stress scales (DASS-21): Psychometric analysis across four racial groups. Anxiety, Stress, and Coping, 20, 253-265.

Quek T. C., Ho C. S., Choo C. C., Nguyen L. H., Tran B. X., \& Ho R. C. (2018). Misophonia in Singaporean psychiatric patients: A cross-sectional study. International Journal of Environmental Research and Public Health, 15(7), 1410. https://doi.org/10.3390/ ijerph15071410.

Ronk, F. R., Korman, J. R., Hooke, G. R., \& Page, A. C. (2013). Assessing clinical significance of treatment outcomes using the DASS-21. Psychological Assessment, 25, 1-8.

Salo, J. (2020). China orders 1,400 military doctors, nurses to treat coronavirus. New York: New York Times. Retrieved March 22, 2020, from https://nypost.com/2020/02/02/china-orders-14000military-doctors-nurses-to-treat-coronavirus/.

Schlanger, Z. (2020). Begging for thermometers, body bags, and gowns: US health care workers are dangerously ill-equipped to fight COVID-19. Retrieved June 20, 2020, from https://time.com/ 5823983/coronavirus-ppe-shortage/.

Steelfisher, G. K., Blendon, R. J., Bekheit, M. M., \& Lubell, K. (2010). The public's response to the $2009 \mathrm{H} 1 \mathrm{~N} 1$ influenza pandemic. New England Journal of Medicine, 362, e65.

Tan, B. Y. Q., Chew, N. W. S., Lee, G. K. H., et al. (2020a). Psychological impact of the COVID-19 pandemic on health care workers in Singapore. Annals of Internal Medicine, 173, 317-320.

Tan, W. H. F., Mcintyre, R. S., Jiang, L., et al. (2020b). Is returning to work during the COVID-19 pandemic stressful? A study on immediate mental health status and psychoneuroimmunity prevention measures of Chinese workforce. Brain, Behavior, and Immunity, 87, 84-92.

Tran, B. X., Dang, A. K., Thai, P. K., et al. (2020a). Coverage of health information by different sources in communities: Implication for COVID-19 epidemic response. International Journal of Environmental Research and Public Health, 17, 1-13.

Tran, B. X., Ha, G. H., Nguyen, L. H., et al. (2020b). Studies of novel coronavirus disease 19 (COVID-19) pandemic: A global analysis of literature. Internation Journal of Environmental Research and Public Health, 17, 1-16.

Tran, B. X., Phan, H. T., Nguyen, T. P. T., et al. (2020c). Reaching further by Village Health Collaborators: The informal health taskforce of Vietname for COVID-19 responses. Journal of Global Health, 10, 1-4.

Wang, C., Pan, R., Wan, X., et al. (2020a). Immediate psychological responses and associated factors during initial stage of the 2019 Coronavirus Disease (COVID-19) epidemic among the general population in China. International Journal of Environmental Research and Public Health, 17, 1-26.

Wang, C., Pan, R., Wan, X., et al. (2020b). A longitudinal study on the mental health of general population during the COVID-19 epidemic in China. Brain, Behavior, and Immunity., 87, 40-48.

Wang, Y., Di, Y., Ye, J., \& Wei, W. (2020c). Study on the public psychological states and its related factors during the outbreak of coronavirus disease 2019 (COVID-19) in some regions of China. Psychology, Health, and Medicine, 26, 13-22.

Zhai, Z. (2020). Facial mask: A necessity to beat COVID-19. Building and Environment, 175, 106827.

Zhang, M. W., Ho, C. S., Fang, F., Lu, Y., \& Ho, R. C. (2014a). Usage of social media and smartphone application in assessment of physical and psychological well-being of individuals in times of a major air pollution crisis. Journal of Medical Internet Research mHealth and uHealth, 2, e16.

Zhang, M. W. B., Ho, C. S. H., Fang, P., Lu, Y., \& Ho, R. C. (2014b). Methodology of developing a smartphone application for crisis research and its clinical application. Technology and Health Care, $22,547-559$.

Publisher's Note Springer Nature remains neutral with regard to jurisdictional claims in published maps and institutional affiliations. 